\title{
On Kazhdan-Lusztig cells in type $B$
}

\author{
Cédric Bonnafé
}

Received: 14 January 2009 / Accepted: 19 May 2009 / Published online: 11 June 2009

(C) Springer Science+Business Media, LLC 2009

\begin{abstract}
We prove that, for any choice of parameters, the Kazhdan-Lusztig cells of a Weyl group of type $B$ are unions of combinatorial cells (defined using the domino insertion algorithm).
\end{abstract}

Keywords Weyl group · Type B · Kazhdan-Lusztig theory · Unequal parameters · Cells

Let $\left(W_{n}, S_{n}\right)$ be the Weyl group of type $B_{n}$, where $S_{n}=\left\{t, s_{1}, \ldots, s_{n-1}\right\}$ and where the Dynkin diagram is given by

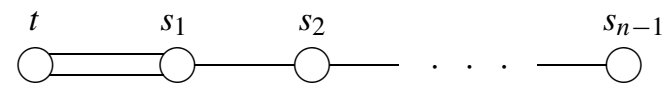

Let $\ell: W_{n} \rightarrow \mathbb{N}=\{0,1,2,3, \ldots\}$ be the length function. Let $\Gamma$ be a totally ordered abelian group and let $\varphi: W_{n} \rightarrow \Gamma$ be a weight function (in the sense of Lusztig [11, $\S 3.1])$. We set

$$
\varphi(t)=b \quad \text { and } \quad \varphi\left(s_{1}\right)=\cdots=\varphi\left(s_{n-1}\right)=a .
$$

To this datum, the Kazhdan-Lusztig theory (with unequal parameters [11]) associates a partition of $W_{n}$ into left, right or two-sided cells [11, Chapter 8].

In [3, Conjectures A and B], Geck, Iancu, Lam and the author have proposed several conjectures for describing these partitions (at least whenever $a, b>0$, but this is not such a big restriction, as can be seen from [2, Corollary 5.8]): they involve a domino insertion algorithm. Roughly speaking, one can define a partition of $W_{n}$ into

The author is partly supported by the ANR (Project No JC07-192339).

C. Bonnafé $(\bowtie)$

Labo. de Math. de Besançon (CNRS: UMR 6623), Université de Franche-Comté, 16 Route de Gray, 25030 Besançon Cedex, France

e-mail: cedric.bonnafe@univ-fcomte.fr 
combinatorial (left, right or two-sided) ( $a, b)$-cells (which depend on $a, b$ and which are defined combinatorially using the domino insertion algorithm): the combinatorial (left, right or two-sided) cells should coincide with the Kazhdan-Lusztig (left, right or two-sided) cells. The aim of this paper is to prove one of the two inclusions (see Theorem 1.5):

Theorem If two elements of $W_{n}$ lie in the same combinatorial (left, right or twosided) cell, then they lie in the same Kazhdan-Lusztig (left, right or two-sided) cell.

In the case of the symmetric group, the partition into left cells (obtained by Kazhdan and Lusztig [8, Theorem 1.4]) uses the Robinson-Schensted correspondence, and the key tool is a description of this correspondence using plactic/coplactic relations (also called Knuth relations). For $W_{n}$, whenever $b>(n-1) a$, the partition into left, right or two-sided cells was obtained by Iancu and the author (see [4, Theorem 7.7] and [1, Corollaries 3.6 and 5.2]) again by using the translation of a generalised Robinson-Schensted correspondence through plactic/coplactic relations.

Recently, M. Taskin [14] and T. Pietraho [12] have independently provided plactic/coplactic relations for the domino insertion algorithm. Our methods rely heavily on their results: we show that, if two elements of $W_{n}$ are directly related by a plactic relation, then they are in the same Kazhdan-Lusztig cell. The key step will be the Propositions 2.10 and 2.11, where some multiplications between elements of the Kazhdan-Lusztig basis are computed by brute force. We then derive some consequences (see Propositions 4.1 and 6.1), where it is proved that some elements are in the same left cells. Then, the rest of the proof just uses the particular combinatorics of Weyl groups of type $B$, together with classical properties of Kazhdan-Lusztig cells.

There are many conjectures concerned with the partition of a general Coxeter group into cells. For finite Coxeter groups in the equal parameter case, most of them were proved by Lusztig. In type $F_{4}$ with arbitrary parameters, they have been checked by Geck [6]. As a consequence of our main theorem, we can complete the proof of some of them for finite Coxeter groups:

Corollary If $(W, S)$ is a finite Coxeter system and if $\varphi: W \rightarrow \Gamma$ is a weight function, then:

(a) Every left cell contains an involution.

(b) If $x \in W$, then $x \sim_{L R} x^{-1}$.

\section{Notation}

\subsection{Weyl group}

Let $\left(W_{n}, S_{n}\right)$ be the Weyl group of type $B_{n}$, where $S_{n}=\left\{t, s_{1}, \ldots, s_{n-1}\right\}$ and where the Dynkin diagram is given by

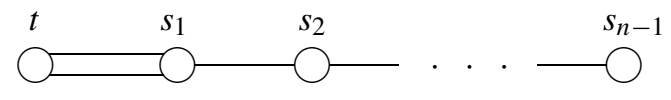


Let $\ell: W_{n} \rightarrow \mathbb{N}=\{0,1,2,3, \ldots\}$ be the length function. Let $I_{n}=\{ \pm 1, \ldots, \pm n\}$ : we shall identify $W_{n}$ with the group of permutations $w$ of $I_{n}$ such that $w(-i)=-w(i)$ for all $w \in I_{n}$. The identification is through the following map

$$
t \longmapsto(1,-1) \quad \text { and } \quad s_{i} \longmapsto(i, i+1)(-i,-i-1) .
$$

The next notation comes from [4, §4]: it is rather technical but will be used throughout this paper. We set $t_{1}=r_{1}=t$ and, for $1 \leqslant i \leqslant n-1$, we set

$$
r_{i+1}=s_{i} r_{i} \quad \text { and } \quad t_{i+1}=s_{i} t_{i} s_{i}
$$

We shall often use the following well-known lemma:

Lemma 1.1 Let $w \in W_{n}, i \in\{1,2, \ldots, n-1\}$ and $j \in\{1,2, \ldots, n\}$. Then:

(a) $\ell\left(w s_{i}\right)>\ell(w)$ (that is, $\left.w s_{i}>w\right)$ if and only if $w(i)<w(i+1)$.

(b) $\ell\left(w t_{j}\right)>\ell(w)$ if and only if $w(j)>0$.

As a permutation of $I_{n}$, we have

$$
t_{i}=(i,-i)
$$

and

$$
r_{i}(j)= \begin{cases}-i & \text { if } j=1 \\ j-1 & \text { if } 2 \leqslant j \leqslant i \\ j & \text { if } i+1 \leqslant j \leqslant n .\end{cases}
$$

An easy computation shows that, if $j \in\{1,2, \ldots, n-1\}$ and $i \in\{1,2, \ldots, n\}$, then

$$
s_{j} r_{i}= \begin{cases}r_{i} s_{j} & \text { if } j>i \\ r_{i+1} & \text { if } j=i \\ r_{i-1} & \text { if } j=i-1 \\ r_{i} s_{j+1} & \text { if } 1 \leqslant j<i-1\end{cases}
$$

Note also that, if $l \geqslant 2$, then

$$
r_{l} r_{l}=r_{l-1} r_{l} s_{1}
$$

We set $a_{0}=1$ and, if $0 \leqslant l \leqslant n$, we set

$$
a_{l}=r_{1} r_{2} \cdots r_{l}
$$

As a permutation of $I_{n}$, we have

$$
a_{l}(i)= \begin{cases}i-1-l & \text { if } 1 \leqslant i \leqslant l, \\ i & \text { if } l+1 \leqslant i \leqslant n .\end{cases}
$$

In particular,

$$
a_{l}^{-1}=a_{l}
$$


and, if $i \in\{1,2, \ldots, n-1\} \backslash\{l\}$, then

$$
a_{l} s_{i} a_{l}= \begin{cases}s_{l-i} & \text { if } i<l \\ s_{i} & \text { if } i>l .\end{cases}
$$

Note also that

$$
\ell\left(a_{l}\right)=\frac{l(l+1)}{2} .
$$

We shall identify the symmetric group $\mathfrak{S}_{n}$ with the subgroup of $W_{n}$ generated by $s_{1}, \ldots, s_{n-1}$. We also set $I_{n}^{+}=\{1,2, \ldots, n\}$. Then, as a group of permutations of $I_{n}$, we have

$$
\mathfrak{S}_{n}=\left\{w \in W_{n} \mid w\left(I_{n}^{+}\right)=I_{n}^{+}\right\} .
$$

If $1 \leqslant i \leqslant j \leqslant n$, we denote by $[i, j]$ the set $\{i, i+1, \ldots, j\}$ and by $\mathfrak{S}_{[i, j]}$ the subgroup of $W_{n}$ (or of $\mathfrak{S}_{n}$ ) generated by $s_{i}, s_{i+1}, \ldots, s_{j-1}$. If $j<i$, then we set $[i, j]=\varnothing$ and $\sigma_{[i, j]}=1$. As a group of permutations of $I_{n}$, we have

$$
\mathfrak{S}_{[i, j]}=\left\{w \in \mathfrak{S}_{n} \mid \forall k \in I_{n}^{+} \backslash[i, j], w(k)=k\right\} .
$$

The longest element of $W_{n}$ will be denoted by $w_{n}$ (it is usually denoted by $w_{0}$, but since we shall use induction on $n$, we need to emphasize its dependence on $n$ ). We denote by $\sigma_{n}$ the longest element of $\mathfrak{S}_{n}$. The longest element of $\mathfrak{S}_{[i, j]}$ will be denoted by $\sigma_{[i, j]}$. As a permutation of $I_{n}$, we have

$$
w_{n}=(1,-1)(2,-2) \cdots(n,-n) .
$$

Note also that

$$
\left\{\begin{array}{l}
w_{n}=t_{1} t_{2} \cdots t_{n}=t_{n} \cdots t_{2} t_{1} \\
w_{n}=a_{n} \sigma_{n}=\sigma_{n} a_{n} \\
\sigma_{n}=\sigma_{[1, n]}
\end{array}\right.
$$

and that

$$
w_{n} \text { is central in } W_{n} \text {. }
$$

\subsection{Decomposition of elements of $W_{n}$}

If $0 \leqslant l \leqslant n$, we denote by $\mathfrak{S}_{l, n-l}$ the subgroup of $\mathfrak{S}_{n}$ generated by $\left\{s_{1}, \ldots, s_{n-1}\right\} \backslash$ $\left\{s_{l}\right\}$. Then $\mathfrak{S}_{l, n-l}=\mathfrak{S}_{[1, l]} \times \mathfrak{S}_{[l+1, n]} \simeq \mathfrak{S}_{l} \times \mathfrak{S}_{n-l}$. We denote by $Y_{l, n-l}$ the set of elements $w \in \mathfrak{S}_{n}$ which are of minimal length in $w \mathfrak{S}_{l, n-l}$. Note that $a_{l}$ normalizes $\mathfrak{S}_{l, n-l}$ (this follows from 1.7).

If $w \in W_{n}$, we denote by $\ell_{t}(w)$ the number of occurrences of $t$ in a reduced decomposition of $w$ (this does not depend on the choice of the reduced decomposition). We set $\ell_{s}(w)=\ell(w)-\ell_{t}(w)$. 
Lemma 1.2 Let $w \in W_{n}$. Then there exists a unique quadruple $(l, \alpha, \beta, \sigma)$ where $0 \leqslant l \leqslant n, \alpha, \beta \in Y_{l, n-l}$ and $\sigma \in \mathfrak{S}_{l, n-l}$ are such that $w=\alpha a_{l} \sigma \beta^{-1}$. Moreover, there exists a unique sequence $1 \leqslant i_{1}<i_{2}<\cdots<i_{l} \leqslant n$ such that $\alpha a_{l}=r_{i_{1}} r_{i_{2}} \cdots r_{i_{l}}$. We have

$$
\begin{gathered}
\ell(w)=\ell(\alpha)+\ell\left(a_{l}\right)+\ell(\sigma)+\ell(\beta), \\
\ell_{t}(w)=l
\end{gathered}
$$

and

$$
\left\{i_{1}, \ldots, i_{l}\right\}=\left\{i \in[1, n] \mid w^{-1}(i)<0\right\}
$$

Note also that

$$
\ell(\alpha)=\sum_{k=1}^{l}\left(i_{k}-k\right)
$$

Proof See [4, Sect. 4, and especially Proposition 4.10].

If $l \in[0, n]$ and if $1 \leqslant i_{1}<\cdots<i_{l} \leqslant n$ and $1 \leqslant j_{1}<\cdots<j_{n-l} \leqslant n$ are two sequences such that $[1, n]=\left\{i_{1}, \ldots, i_{l}\right\} \cup\left\{j_{1}, \ldots, j_{n-l}\right\}$, then it follows easily from 1.2 that

$$
\begin{cases}\left(r_{i_{1}} \cdots r_{i_{l}}\right)^{-1}\left(i_{k}\right)=k-l-1 & \text { if } 1 \leqslant k \leqslant l, \\ \left(r_{i_{1}} \cdots r_{i_{l}}\right)^{-1}\left(j_{k}\right)=l+k & \text { if } 1 \leqslant k \leqslant n-l .\end{cases}
$$

The elements $\alpha, \beta$ and $\sigma$ of the previous lemma will we denoted by $\alpha_{w}, \beta_{w}$ and $\sigma_{w}$ respectively. We have

$$
\ell_{t}\left(w^{-1}\right)=\ell_{t}(w), \quad \alpha_{w^{-1}}=\beta_{w}, \quad \beta_{w^{-1}}=\alpha_{w} \quad \text { and } \quad \sigma_{w^{-1}}=a_{l}\left(\sigma_{w}\right)^{-1} a_{l} .
$$

We shall now describe how the multiplication by the longest element $w_{n}$ acts on the decomposition given by Lemma 1.2. For this, we denote by $\sigma_{l, n-l}$ the longest element of $\mathfrak{S}_{l, n-l}$.

Proposition 1.3 Let $w \in W_{n}$ and let $l=\ell_{t}(w)$. Then:

(a) $\ell_{t}\left(w_{n} w\right)=n-l$.

(b) $\alpha_{w_{n} w}=\alpha_{w} \sigma_{n} \sigma_{n-l, l}$ and $\beta_{w_{n} w}=\beta_{w} \sigma_{n} \sigma_{n-l, l}$.

(c) $\sigma_{w_{n} w}=\sigma_{n} \sigma \sigma_{n}^{-1} \sigma_{n-l, l}$.

(d) Let $1 \leqslant i_{1}<\cdots<i_{l} \leqslant n$ be the sequence such that $\alpha_{w} a_{l}=r_{i_{1}} \cdots r_{i_{l}}$. Then $\alpha_{w_{n} w}=r_{j_{1}} \cdots r_{j_{n-l}}$, where $1 \leqslant j_{1}<\cdots<j_{n-l} \leqslant n$ is the sequence such that $\left\{i_{1}, \ldots, i_{l}\right\} \cup\left\{j_{1}, \ldots, j_{n-l}\right\}=[1, n]$.

Proof (a) is clear. (d) follows from Lemma 1.2. We now prove (b) and (c) simultaneously. For this, let $\alpha^{\prime}=\alpha_{w} \sigma_{n} \sigma_{n-l, l}, \beta^{\prime}=\beta_{w} \sigma_{n} \sigma_{n-l, l}$ and $\sigma^{\prime}=\sigma_{n} \sigma_{w} \sigma_{n}^{-1} \sigma_{n-l, l}$. By the unicity statement of the Lemma 1.2 , we only need to show the following three properties: 
(1) $\alpha^{\prime}, \beta^{\prime} \in Y_{n-l, l}$.

(2) $\sigma^{\prime} \in \mathfrak{S}_{n-l, l}$.

(3) $w_{n} w=\alpha^{\prime} a_{n-l} \sigma^{\prime} \beta^{\prime-1}$.

For this, note first

$$
\sigma_{n} \mathfrak{S}_{l, n-l} \sigma_{n}^{-1}=\mathfrak{S}_{n-l, l},
$$

so that (2) follows immediately. This also implies that $\sigma_{n} \sigma_{n-l, l} \sigma_{n}^{-1}=\sigma_{l, n-l}$ because conjugacy by $\sigma_{n}$ in $\mathfrak{S}_{n}$ preserves the length.

Let us now show (1). Let $i \in\{1,2, \ldots, n\} \backslash\{n-l\}$. We want to show that $\ell\left(\alpha^{\prime} s_{i}\right)>\ell\left(\alpha^{\prime}\right)$. By Lemma 1.1, this amounts to show that $\alpha^{\prime}(i+1)>\alpha^{\prime}(i)$. But $\alpha^{\prime}=\alpha_{w} \sigma_{l, n-l} \sigma_{n}$. Also $\sigma_{n}(i)=n+1-i>\sigma_{n}(i+1)=n-i$ and $n+1-i$ and $n-i$ both belong to the same interval $[1, l]$ or $[l+1, n]$. Hence $\sigma_{l, n-l} \sigma_{n}(i)<$ $\sigma_{l, n-l} \sigma_{n}(i+1)$ and $\alpha_{w} \sigma_{l, n-l} \sigma_{n}(i)<\alpha_{w} \sigma_{l, n-l} \sigma_{n}(i+1)$ since $\alpha_{w} \in Y_{l, n-l}$. This shows that $\alpha^{\prime} \in Y_{n-l, l}$. Similarly, $\beta^{\prime} \in Y_{n-l, l}$. So (1) is proved.

It remains to show (3). We have

$$
\begin{aligned}
\alpha^{\prime} a_{n-l} \sigma^{\prime} \beta^{\prime-1} & =\left(\alpha_{w} \sigma_{n} \sigma_{n-l, l}\right) \cdot a_{n-l} \cdot\left(\sigma_{n} \sigma_{w} \sigma_{n}^{-1} \sigma_{n-l, l}\right) \cdot\left(\sigma_{n-l, l}^{-1} \sigma_{n}^{-1} \beta_{w}^{-1}\right) \\
& =\alpha_{w} \sigma_{n} \sigma_{n-l, l} a_{n-l} \sigma_{n} \sigma_{w} \beta_{w}^{-1}
\end{aligned}
$$

But $\sigma_{n-l, l}=\sigma_{[n-l+1, n]} \sigma_{n-l}$ and $\sigma_{n} \sigma_{[n-l+1, n]} \sigma_{n}^{-1}=\sigma_{[1, l]}=\sigma_{l}$ so

$$
\begin{aligned}
\alpha^{\prime} a_{n-l} \sigma^{\prime} \beta^{\prime-1} & =\alpha_{w} \sigma_{l} \sigma_{n} \sigma_{n-l} a_{n-l} \sigma_{n}^{-1} \sigma_{w} \beta_{w}^{-1} \\
& =\alpha_{w} \sigma_{l} \sigma_{n} w_{n-l} \sigma_{n}^{-1} \sigma_{w} \beta_{w}^{-1},
\end{aligned}
$$

the last equality following from 1.12. Now, $\sigma_{n} w_{n-l} \sigma_{n}^{-1}=w_{l} w_{n}$ (see again 1.12) so

$$
\begin{aligned}
\alpha^{\prime} a_{n-l} \sigma^{\prime} \beta^{\prime-1} & =\alpha_{w} \sigma_{l} w_{l} w_{n} \sigma_{w} \beta_{w}^{-1} \\
& =\alpha_{w} a_{l} w_{n} \sigma_{w} \beta_{w}^{-1} \\
& =w_{n} \alpha_{w} a_{l} \sigma_{w} \beta_{w}^{-1}=w_{n} w
\end{aligned}
$$

the second equality following from 1.12 and the third one from the fact that $w_{n}$ is central (see 1.13).

\subsection{Subgroups $W_{m}$ of $W_{n}$}

If $m \leqslant n$, we shall view $W_{m}$ naturally as a subgroup of $W_{n}$ (the pointwise stabilizer of $[m+1, n])$. It is the standard parabolic subgroup generated by $S_{m}=\left\{t, s_{1}, \ldots, s_{m-1}\right\}$ : we denote by $X_{n}^{(m)}$ the set of $w \in W_{n}$ which are of minimal length in $w W_{m}$. For simplification, we set $X_{n}=X_{n}^{(n-1)}$. It follows from Lemma 1.1 that:

Lemma 1.4 Let $w$ be an element of $W_{n}$. Then $w$ belongs to $X_{n}^{(m)}$ if and only if $0<w(1)<w(2)<\cdots<w(m)$. 
If $I=\left\{i_{1}, \ldots, i_{l}\right\} \subseteq[1, n-1]$ with $i_{1}<\cdots<i_{l}$, then we set

$$
c_{I}=s_{i_{1}} s_{i_{2}} \cdots s_{i_{l}} \quad \text { and } \quad d_{I}=s_{i_{l}} \cdots s_{i_{2}} s_{i_{1}} .
$$

By convention, $c_{\varnothing}=d_{\varnothing}=1$. We have

$$
X_{n}=\left\{c_{[i, n-1]} \mid 1 \leqslant i \leqslant n\right\} \dot{\cup}\left\{d_{[1, i]} t c_{[1, n-1]} \mid 0 \leqslant i \leqslant n-1\right\} .
$$

\subsection{Hecke algebra}

We fix a totally ordered abelian group $\Gamma$ (denoted additively) and a weight function $\varphi: W_{n} \rightarrow \Gamma$. We set

$$
\varphi(t)=b \quad \text { and } \quad \varphi\left(s_{1}\right)=a \quad\left(=\varphi\left(s_{2}\right)=\cdots=\varphi\left(s_{n-1}\right)\right) .
$$

Note that

$$
\varphi(w)=\ell_{t}(w) b+\ell_{s}(w) a
$$

for all $w \in W_{n}$.

We denote by $A$ the group algebra $\mathbb{Z}[\Gamma]$. We shall use an exponential notation: $A=\underset{\gamma \in \Gamma}{\oplus} \mathbb{Z} e^{\gamma}$, where $e^{\gamma} \cdot e^{\gamma^{\prime}}=e^{\gamma+\gamma^{\prime}}$ for all $\gamma, \gamma^{\prime} \in \Gamma$. We set

$$
Q=e^{b} \quad \text { and } \quad q=e^{a} .
$$

Note that $Q$ and $q$ are not necessarily algebraically independent. We set

$$
A_{<0}=\underset{\gamma<0}{\bigoplus} \mathbb{Z} e^{\gamma}
$$

and we define similarly $A_{\leqslant 0}, A_{>0}$ and $A_{\geqslant 0}$.

We shall denote by $\mathcal{H}_{n}$ the Hecke algebra of $W_{n}$ with parameter $\varphi$ : it is the free $A$-module with basis $\left(T_{w}\right)_{w \in W_{n}}$ and the multiplication is $A$-bilinear and is completely determined by the following rules:

$$
\begin{cases}T_{w} T_{w^{\prime}}=T_{w w^{\prime}} & \text { if } \ell\left(w w^{\prime}\right)=\ell(w)+\ell\left(w^{\prime}\right), \\ \left(T_{t}-Q\right)\left(T_{t}+Q^{-1}\right)=0, & \\ \left(T_{s_{i}}-q\right)\left(T_{s_{i}}+q^{-1}\right)=0 & \text { if } 1 \leqslant i \leqslant n-1 .\end{cases}
$$

We also set

$$
\mathcal{H}_{n}^{<0}=\underset{w \in W_{n}}{\oplus} A_{<0} T_{w}
$$

Finally, we denote by ${ }^{-}: \mathcal{H}_{n} \rightarrow \mathcal{H}_{n}$ the unique $A$-semilinear involution of $\mathcal{H}_{n}$ such that $\overline{e^{\gamma}}=e^{-\gamma}$ and $\bar{T}_{w}=T_{w^{-1}}^{-1}$ for all $\gamma \in \Gamma$ and $w \in W_{n}$. 


\subsection{Kazhdan-Lusztig basis}

We shall recall here the basic definitions of Kazhdan-Lusztig theory. If $w \in W_{n}$, then, by [11, Theorem 5.2], there exists a unique $C_{w} \in \mathcal{H}_{n}$ such that

$$
\left\{\begin{array}{l}
\bar{C}_{w}=C_{w} \\
C_{w} \equiv T_{w} \quad \bmod \mathcal{H}_{n}^{<0} .
\end{array}\right.
$$

Note that $[11, \S 5.3]$

$$
C_{w}-T_{w} \in \underset{w^{\prime}<w}{\oplus} A_{<0} T_{w^{\prime}},
$$

where $\leqslant$ denotes the Bruhat order on $W_{n}$. In particular, $\left(C_{w}\right)_{w \in W_{n}}$ is an $A$-basis of $\mathcal{H}_{n}$, called the Kazhdan-Lusztig basis of $\mathcal{H}_{n}$.

\subsection{Cells}

If $x, y \in W_{n}$, then we shall write $x \stackrel{L}{\longleftarrow} y$ (resp. $x \stackrel{R}{\longleftarrow} y$, resp. $x \stackrel{L R}{\longleftarrow} y$ ) if there exists $h \in \mathcal{H}_{n}$ such that the coefficient of $C_{x}$ in the decomposition of $h C_{y}$ (resp. $C_{y} h$, resp. $h C_{y}$ or $\left.C_{y} h\right)$ is non-zero. We denote by $\leqslant_{L}$ (resp. $\leqslant_{R}$, resp. $\left.\leqslant_{L R}\right)$ the transitive closure of $\stackrel{L}{\longleftarrow}$ (resp. $\stackrel{R}{\longleftarrow}$, resp. $\stackrel{L R}{\longleftarrow}$ ). Then $\leqslant_{L}, \leqslant_{R}$ and $\leqslant_{L R}$ are preorders on $W_{n}$ and we denote respectively by $\sim_{L}, \sim_{R}$ and $\sim_{L R}$ the associated equivalence relations [11, Chapter 8]. An equivalence class for $\sim_{L}$ (resp. $\sim_{R}$, resp. $\sim_{L R}$ ) is called a left (resp. right, resp. two-sided) cell. We recall the following result [11, §8.1]: if $x$, $y \in W_{n}$, then

$$
x \sim_{L} y \Longleftrightarrow x^{-1} \sim_{R} y^{-1}
$$

\subsection{Domino insertion}

If $r \geqslant 0$ and $w \in W_{n}$, then the domino insertion algorithm (see [9], [15], [16]) into the 2-core $\delta_{r}=(r, r-1, \ldots, 2,1)$ associates to $w$ a standard domino tableau $D_{r}(w)$ (with $n$ dominoes, filled with $\{1,2, \ldots, n\}$ ). If $D$ is a domino tableau, we denote by $\mathbf{s h}(D)$ its shape: we shall denote by $\mathbf{s h}_{r}(w)$ the shape of $D_{r}(w)$ (which is equal to the shape of $D_{r}\left(w^{-1}\right)$, loc. cit.).

If $x$ and $y \in W_{n}$ we shall write $x \sim_{L}^{r} y$ (resp. $x \sim_{R}^{r} y$, resp. $x \sim_{L R}^{r} y$ ) if $D_{r}\left(x^{-1}\right)=D_{r}\left(y^{-1}\right)\left(\right.$ resp. $D_{r}(x)=D_{r}(y)$, resp. $\left.\mathbf{s h}_{r}(x)=\mathbf{s h}_{r}(y)\right)$. These are equivalence relations on $W_{n}$. Note that $\sim_{L R}^{r}$ is the equivalence relation generated by $\sim_{L}^{r}$ and $\sim_{R}^{r}$.

We denote by $\approx_{L}^{r+1}$ (resp. $\approx_{R}^{r+1}$, resp. $\approx_{L R}^{r+1}$ ) the equivalence relation generated by $\sim_{L}^{r}$ and $\sim_{L}^{r+1}$ (resp. $\sim_{R}^{r}$ and $\sim_{R}^{r+1}$, resp. $\sim_{L R}^{r}$ and $\sim_{L R}^{r+1}$ ). Recall the following conjecture from [3, Conjectures $\mathrm{A}$ and $\mathrm{B}]$ :

Conjecture. Assume that $a, b>0$. Let $r \geqslant 0$ and $? \in\{L, R, L R\}$.

(a) If $r a<b<(r+1) a$, then the relations $\sim_{\text {? }}$ and $\sim_{\text {? }}^{r}$ coincide.

(b) If $r \geqslant 1$ and $b=r a$, then the relations $\sim_{\text {? }}$ and $\approx_{\text {? }}^{r}$ coincide. 
The main result of this paper is the following partial result towards the previous conjecture:

Theorem 1.5 Assume that $a, b>0$. Let $r \geqslant 0, ? \in\{L, R, L R\}$ and $x, y \in W_{n}$. Then:

(a) If $r a<b<(r+1) a$ and $x \sim_{\text {? }}^{r} y$, then $x \sim$ ? $y$.

(b) If $r \geqslant 1, b=$ ra and $x \approx_{\text {? }}^{r} y$, then $x \sim_{\text {? }} y$.

The other sections of this paper are devoted to the proof of this theorem.

Comments If one assumes Lusztig's Conjectures P1, P2,.., P15 in [11, Chapter 14], then Theorem 1.5 implies that the statement (a) of the Conjecture is true. Indeed, Lusztig's Conjectures imply in this case that the left cell representations are irreducible, and one can conclude by a counting argument.

Recently, Pietraho [13] proved that, if one assumes the above Lusztig's Conjectures, then both statement (a) and (b) hold and the representations carried by the left cells are the ones we expect.

In the case where $b>(n-1) a$, Theorem 1.5 was proved in [4, Theorem 7.7] (in fact, the conjecture was also proved) by using a counting argument. The proof here does not make use of the counting argument.

\section{Kazhdan-Lusztig polynomials, structure constants}

Hypothesis and notation. From now on, and until the end of this paper, we assume that $a, b$ are positive. Recall that $Q=e^{b}$ and $q=e^{a}$, so that $\mathbb{Z}\left[Q, Q^{-1}, q, q^{-1}\right] \subseteq A$. If $p \in A$, we denote by $\tau_{A}(p)$ the coefficient of $1=e^{0}$ in the expansion of $p$ in the basis $\left(e^{\gamma}\right)_{\gamma \in \Gamma \text {. }}$.

\subsection{Recollection of general facts}

If $x$ and $y$ are elements of $W_{n}$, we set

$$
C_{x} C_{y}=\sum_{z \in W_{n}} h_{x, y, z} C_{z},
$$

where the $h_{x, y, z}$ belong to $A$ and satisfy

$$
\overline{h_{x, y, z}}=h_{x, y, z} \text {. }
$$

We also set

$$
C_{y}=\sum_{x \in W_{n}} p_{x, y}^{*} T_{x} \quad \text { and } \quad p_{x, y}=e^{\varphi(y)-\varphi(x)} p_{x, y}^{*} .
$$


Recall [11, Proposition 5.4] that

$$
\begin{cases}p_{y, y}^{*}=p_{y, y}=1, & \\ p_{x, y}^{*} \in A_{<0} & \text { if } x \neq y . \\ p_{x, y}^{*}=p_{x, y}=0 & \text { if } x \neq y, \\ p_{x, y} \in A_{\geqslant 0}, & \\ \tau_{A}\left(p_{x, y}\right)=1 & \text { if } x \leqslant y .\end{cases}
$$

Now, if $s \in S_{n}$, Lusztig [11, Proposition 6.3] has defined inductively a family of polynomials $\left(M_{x, y}^{s}\right)_{s x<x<y<s y}$ by the following properties:

$$
\begin{gathered}
\overline{M_{x, y}^{s}}=M_{x, y}^{s}, \\
M_{x, y}^{s}+\sum_{\substack{x<z<y \\
s z<z}} p_{x, z}^{*} M_{z, y}^{s}-e^{\varphi(s)} p_{x, y}^{*} \in A_{<0} .
\end{gathered}
$$

With this notation, we have [11, Theorem 6.6]:

Theorem 2.1 (Kazhdan-Lusztig, Lusztig) Let $s \in S_{n}$ and let $y \in W_{n}$. Then:

(a) $C_{s} C_{y}= \begin{cases}C_{s y}+\sum_{s x<x<y} M_{x, y}^{s} C_{x} & \text { if } s y>y, \\ \left(e^{\varphi(s)}+e^{-\varphi(s)}\right) C_{y} & \text { if } s y<y .\end{cases}$

(b) If $s y<y$, and if $x \leqslant y$, then

$$
p_{x, y}= \begin{cases}q^{2} p_{x, s y}+p_{s x, s y}-\sum_{\substack{x \leqslant z<s y \\ s z<z}} e^{\varphi(y)-\varphi(z)} p_{x, z} M_{z, s y}^{s} & \text { if } s x<x, \\ p_{s x, y} & \text { if } s x>x,\end{cases}
$$

and

$$
p_{x, y}^{*}= \begin{cases}q p_{x, s y}^{*}+p_{s x, s y}^{*}-\sum_{\substack{x \leqslant z<s y \\ s z<z}} p_{x, z}^{*} M_{z, s y}^{s} & \text { if } s x<x, \\ e^{-\varphi(s)} p_{s x, y}^{*} & \text { if } s x>x .\end{cases}
$$

Corollary 2.2 If $s, s^{\prime} \in\left\{s_{1}, \ldots, s_{n-1}\right\}$ and $x, y \in W_{n}$ are such that $s x<x<s^{\prime} x=$ $y<s y$, then $x \sim_{L} y$.

Proof See [10, Proposition 5 (b)].

\subsection{Special features in type $B$}

The results of the previous subsection hold for any Coxeter group (finite or not). In this subsection, we shall investigate what is implied by the structure of $W_{n}$. The particular ingredient we shall need is the following lemma [4, §4]: 
Lemma $2.3\left\{a_{l} \mid 0 \leqslant l \leqslant n\right\}$ is the set of elements $w \in W_{n}$ which have minimal length in $\mathfrak{S}_{n} w \mathfrak{S}_{n}$. If $x<a_{l}$ for some $l \in\{1,2, \ldots, n\}$ and some $x \in W_{n}$, then $\ell_{t}(x)<\ell_{t}\left(a_{l}\right)=l$.

It has the following consequences (here, if $p \in \mathbb{Z}[q]$, we denote by $\operatorname{deg}_{q} p$ its degree in the variable $q$ ):

Corollary 2.4 Let $x$ and $y$ be two elements of $W_{n}$ such that $x \leqslant y$ and $\ell_{t}(x)=\ell_{t}(y)$. Then:

(a) $p_{x, y} \in \mathbb{Z}[q]$ and, if $x \neq y$, then $\operatorname{deg}_{q} p_{x, y}<\ell(y)-\ell(x)$.

(b) If $1 \leqslant i \leqslant n-1$ is such that $s_{i} x<x<y<s_{i} y$, then $M_{x, y}^{s_{i}} \in \mathbb{Z}$ : it is equal to $\tau_{A}\left(q p_{x, y}^{*}\right)$ (note also that $q p_{x, y}^{*} \in \mathbb{Z}\left[q^{-1}\right]$ ).

Proof We shall prove (a) and (b) together by induction on the pair $(\ell(y), \ell(y)-\ell(x))$ (with lexicographic order). The result is obvious if $\ell(y)=\ell(x)$ or if $\ell(y) \leqslant 1$. So assume now that $\ell(y)>1$, that $\ell(y)-\ell(x)>0$ and that (a) and (b) hold for all pairs $\left(x^{\prime}, y^{\prime}\right)$ such that $\left(\ell\left(y^{\prime}\right), \ell\left(y^{\prime}\right)-\ell\left(x^{\prime}\right)\right)<(\ell(y), \ell(y)-\ell(x))$. First, note that

$$
e^{\varphi(y)-\varphi(x)}=q^{\ell(y)-\ell(x)},
$$

because $\varphi(y)-\varphi(x)=\left(\ell_{t}(y)-\ell_{t}(x)\right) b+\left(\ell_{s}(y)-\ell_{s}(x)\right) a=\left(\ell_{s}(y)-\ell_{s}(x)\right) a=$ $(\ell(y)-\ell(x)) a$.

Let us first prove (a). So we have $x<y$ and $\ell_{t}(x)=\ell_{t}(y)$. By Lemma 2.3, this implies that there exists $i \in\{1,2, \ldots, n-1\}$ such that $s_{i} y<y$ or $y s_{i}<y$. In the second case, one can exchange $y$ and $y^{-1}$ (and $x$ and $x^{-1}$ ) by using $[11, \S 5.6]$, so that we may assume that $s_{i} y<y$. Then, Theorem 2.1 (b) can be rewritten as follows:

$$
p_{x, y}= \begin{cases}\left(q^{2} p_{x, s_{i} y}-q^{\ell(y)-\ell(x)} M_{z, s_{i} y}^{s_{i}}\right)+p_{s_{i} x, s_{i} y} & \\ -\sum_{s_{i} z<z} x<s_{i} y q^{\ell(y)-\ell(z)} p_{x, z} M_{z, s_{i} y}^{s_{i}} & \text { if } s_{i} x<x, \\ p_{s_{i} x, y} & \text { if } s_{i} x>x .\end{cases}
$$

If $s_{i} x>x$, then the result follows from the induction hypothesis. If $s_{i} x<x$, then

$$
q^{2} p_{x, s_{i} y}-q^{\ell(y)-\ell(x)} M_{x, s_{i} y}^{s_{i}}=q^{\ell(y)-\ell(x)}\left(q p_{x, s_{i} y}^{*}-M_{x, s_{i} y}^{s_{i}}\right)
$$

belong to $\mathbb{Z}[q]$ and has degree $<\ell(y)-\ell(x)$ by the induction hypothesis. The other terms in the above formula also belong to $\mathbb{Z}[q]$ and also have degree $<\ell(y)-\ell(x)$ by the induction hypothesis. So we get (a).

Let us now prove (b). So we assume that $s_{i} x<x<y<s_{i} y$. Then, using the induction hypothesis and 2.1, the condition $2.2 \mathrm{~b}$ can be rewritten

$$
M_{x, y}^{s_{i}}-q p_{x, y}^{*} \in A_{<0} .
$$

Now, the result follows easily from (a). 
Now, if $t x<x<y<t y$ are such that $\ell_{t}(x)=\ell_{t}(y)$, let us define an element $\mu_{x, y} \in A$ by induction on $\ell(y)-\ell(x)$ by the following formula:

$$
\mu_{x, y}=p_{x, y}-\sum_{\substack{x<z<y \\ t z<z}} p_{x, z} \mu_{z, y} .
$$

It follows easily from Corollary 2.4 (and an induction argument on $\ell(y)-\ell(x)$ ) that

$$
\mu_{x, y} \in \mathbb{Z}[q] \text { and } \operatorname{deg}_{q} \mu_{x, y}<\ell(y)-\ell(x) .
$$

Moreover:

Corollary 2.5 Assume that $t x<x<y<$ ty and that $\ell_{t}(x)=\ell_{t}(y)$. Then:

(a) If $b>(\ell(y)-\ell(x)) a$, then $M_{x, y}^{t}=Q q^{\ell(x)-\ell(y)} \mu_{x, y}+Q^{-1} q^{\ell(y)-\ell(x)} \overline{\mu_{x, y}}$.

(b) If $b=(\ell(y)-\ell(x)) a$, then $M_{x, y}^{t}=\mu_{x, y}+\overline{\mu_{x, y}}-\tau_{A}\left(\mu_{x, y}\right)$.

Proof Let us assume that $b \geqslant(\ell(y)-\ell(x)) a$. We shall prove the result by induction on $\ell(y)-\ell(x)$. By the induction hypothesis, the condition $2.2 \mathrm{~b}$ can we written

$$
M_{x, y}^{t}-Q q^{\ell(x)-\ell(y)} p_{x, y}+\sum_{\substack{x<z<y \\ t z<z}} p_{x, z}^{*}\left(Q q^{\ell(z)-\ell(y)} \mu_{z, y}+Q^{-1} q^{\ell(y)-\ell(z)} \overline{\mu_{z, y}}\right) \in A_{<0} .
$$

But, if $x<z<y$ and $t z<z$, then

$$
p_{x, z}^{*} Q^{-1} q^{\ell(y)-\ell(z)} \overline{\mu_{z, y}} \in A_{<0}
$$

because $p_{x, z}^{*} \in A_{<0}, \overline{\mu_{z, y}} \in A_{\leqslant 0}$ and $Q^{-1} q^{\ell(y)-\ell(z)}=e^{-b+(\ell(y)-\ell(z)) a} \in A_{<0}$ (since $\ell(y)-\ell(z)<\ell(y)-\ell(x))$. Therefore,

$$
M_{x, y}^{t}-Q q^{\ell(x)-\ell(y)} p_{x, y}+\sum_{\substack{x<z<y \\ t z<z}} Q q^{\ell(x)-\ell(y)} p_{x, z} \mu_{z, y} \in A_{<0} .
$$

In other words,

$$
M_{x, y}^{t}-Q q^{\ell(x)-\ell(y)} \mu_{x, y} \in A_{<0} .
$$

Let $\mu=Q q^{\ell(x)-\ell(y)} \mu_{x, y}$. Two cases may occur:

- If $b>(\ell(y)-\ell(x)) a$, then $\mu \in A_{>0}$ and so the condition 2.2a forces $M_{x, y}^{t}=$ $\mu+\bar{\mu}$, as required.

- If $b=(\ell(y)-\ell(x)) a$, then $\mu=\mu_{x, y} \in A \geqslant 0$ and now the condition 2.2a forces $M_{x, y}^{t}=\mu+\bar{\mu}-\tau_{A}(\mu)$, as required.

The proof of the Corollary is complete.

We conclude this subsection with two results involving the decomposition of Lemma 1.2. 
Lemma 2.6 Let $x$ and $y$ be two elements of $W_{n}$ and let $s \in S_{n}$ be such that $s x<$ $x<y<s y, \ell_{t}(x)=\ell_{t}(y)$ and $\beta_{x}=\beta_{y}=\beta$. Then $M_{x, y}^{s}=M_{x \beta, y \beta}^{s}$ (note that $\beta_{x \beta}=$ $\left.\beta_{y \beta}=1\right)$.

Proof See [4, Proposition 7.2]. Strictly speaking, in [4], the authors are generally working with a special choice of a function $\varphi$ ("asymptotic case"): however, the reader can check that the proof of this particular result, namely [4, Proposition 7.2], remains valid for all choices of parameters.

Proposition 2.7 Let $l \in[0, n]$, let $\sigma$ and $\sigma^{\prime} \in \mathfrak{S}_{l, n-l}$ be such that $\sigma \sim_{L} \sigma^{\prime}$ and let $\beta \in Y_{l, n-l}$. Then

$$
a_{l} \sigma \beta^{-1} \sim_{L} a_{l} \sigma^{\prime} \beta^{-1}
$$

Proof By the description of Kazhdan-Lusztig left cells in the symmetric group [8, Theorem 1.4 and §4], we may assume that there exist two elements $s$ and $s^{\prime}$ in $\left\{s_{1}, \ldots, s_{l-1}, s_{l+1}, \ldots, s_{n-1}\right\}$ such that $\sigma^{\prime}=s^{\prime} \sigma$ and $s \sigma<\sigma<\sigma^{\prime}<s \sigma^{\prime}$. Let $u=a_{l} s a_{l}$ and $u^{\prime}=a_{l} s^{\prime} a_{l}$. Then $u$ and $u^{\prime}$ belong to $\left\{s_{1}, \ldots, s_{l-1}, s_{l+1}, \ldots, s_{n-1}\right\}$ by 1.7, and

$$
u a_{l} \sigma \beta^{-1}<a_{l} \sigma \beta^{-1}<u^{\prime} a_{l} \sigma \beta^{-1}=a_{l} \sigma^{\prime} \beta^{-1}<u a_{l} \sigma^{\prime} \beta^{-1} .
$$

So $(*)$ follows from Corollary 2.2.

\section{$2.3 *$-operation}

We shall recall the definition of the $*$-operation (see $[8, \S 4]$ ) and prove some properties which are particular to the type $B$. Let us introduce some notation. If $1 \leqslant i \leqslant n-2$ and $x \in W_{n}$, we set

$$
\mathcal{R}_{i}(x)=\left\{s \in\left\{s_{i}, s_{i+1}\right\} \mid \ell(x s)<\ell(x)\right\}
$$

We denote by $\mathcal{D}_{i}\left(W_{n}\right)$ the set of $x \in W_{n}$ such that $\left|\mathcal{R}_{i}(x)\right|=1$. If $x \in \mathcal{D}_{i}\left(W_{n}\right)$, then it is readily seen that the set $\left\{x s_{i}, x s_{i+1}\right\} \cap \mathcal{D}_{i}\left(W_{n}\right)$ is a singleton. We shall denote by $\gamma_{i}(x)$ the unique element of this set (it is denoted by $x^{*}$ in $[8, \S 4]$, but we want to emphasize that it depends on $i$ ). Note that

$$
\gamma_{i} \circ \gamma_{i}=\operatorname{Id}_{\mathcal{D}_{i}\left(W_{n}\right)}
$$

We recall Kazhdan-Lusztig result [8, Corollary 4.3]: if $x$ and $y \in \mathcal{D}_{i}\left(W_{n}\right)$, then

$$
x \sim_{L} y \Longleftrightarrow \gamma_{i}(x) \sim_{L} \gamma_{i}(y) .
$$

The fact that $t$ is not conjugate to any of the $s_{k}$ 's implies the following easy fact:

Proposition 2.8 Let $x \in W_{n}$ and let $1 \leqslant k \leqslant n-1$. Then $x s_{k}>x$ if and only if $t x s_{k}>t x$. 
Proof Indeed, by Lemma 1.1, we have $x s_{k}>x$ if and only if $x(k)<x(k+1)$. But, for any $j \in I_{n}^{+}$, there is no element $j^{\prime} \in I_{n}$ such that $t(j)<j^{\prime}<j$. So $x(k)<x(k+1)$ if and only if $t x(k)<t x(k+1)$ that is, if and only if $t x s_{k}>t x$ (again by Lemma 1.1).

The proposition 2.8 implies immediately the following result:

Corollary 2.9 Let $x \in W_{n}$ and let $1 \leqslant i \leqslant n-2$. Then $x \in \mathcal{D}_{i}\left(W_{n}\right)$ if and only if tx $\in \mathcal{D}_{i}\left(W_{n}\right)$. If this is the case, then $\gamma_{i}(t x)=t \gamma_{i}(x)$.

2.4 Two relations $\stackrel{L}{\longleftarrow}$

The crucial steps towards the proof of Theorem 1.5 are the following two propositions, whose proofs will be given in sections 3 and 5 respectively.

Proposition 2.10 Let $l \in\{1, \ldots, n-1\}$ and assume that $b \geqslant(n-1) a$. Then $M_{r_{1} \cdots r_{l} \sigma_{[l+1, n]}, r_{2} \ldots r_{l} r_{n} \sigma_{[l+1, n]}}^{t} \neq 0$.

Proposition 2.11 Let $l \in\{1, \ldots, n-1\}$ and assume that $(n-2) a<b \leqslant(n-1) a$. Then $a_{l-1} \sigma_{[l, n]} \stackrel{L}{\longleftarrow} a_{l} \sigma_{[l, n]}$.

\section{Proof of Proposition 2.10}

Notation. If $u, v \in W_{n}$ are such that $u \leqslant v$, we denote by $[u ; v]$ the Bruhat interval between $u$ and $v$. In this section, and only in this section, we assume that $l \geqslant 1$ and $b \geqslant(n-1) a$ and we set $x=r_{1} \cdots r_{l} \sigma_{[l+1, n]}$ and $y=r_{2} \ldots r_{l} r_{n} \sigma_{[l+1, n]}$.

\subsection{Easy reduction}

Note that

$$
t x<x<y<t y,
$$

so it makes sense to compute $M_{x, y}^{t}$. Moreover, $\ell(y)-\ell(x)=n-1$ so, by Corollary 2.5 , we only need to prove that $\mu_{x, y} \neq 0$ (even if $b=(n-1) a$ ). For this, we only need to show that

$$
\tau_{A}\left(\mu_{x, y}\right) \neq 0
$$

3.2 The Bruhat interval $[x ; y]$

First, note that

$$
x=a_{l} \sigma_{[l+1, n]}=\sigma_{[l+1, n]} a_{l}
$$

and

$$
y=s_{1} \cdots s_{l-1} s_{n-1} \cdots s_{l} x=s_{1} \cdots s_{l-1} s_{n-1} \cdots s_{l} \sigma_{[l+1, n]} a_{l}=c_{[1, l-1]} \sigma_{[l, n]} a_{l} .
$$


Since $a_{l}$ has minimal length in $\mathfrak{S}_{n} a_{l}$, the map

$$
\begin{aligned}
{\left[\sigma_{[l+1, n]} ; c_{[1, l-1]} \sigma_{[l, n]}\right] } & \longrightarrow[x ; y] \\
z & \longmapsto z a_{l}
\end{aligned}
$$

is an increasing bijection [11, Lemma 9.10 (f)]. Since the support of $c_{[1, l-1]}$ is disjoint from the support of $\sigma_{[l, n]}$, the map

$$
\begin{aligned}
{\left[1 ; c_{[1, l]}\right] \times\left[\sigma_{[l+1, n]} ; \sigma_{[l, n]}\right] } & \longrightarrow\left[\sigma_{[l+1, n]} ; c_{[1, l-1]} \sigma_{[l, n]}\right] \\
\left(z, z^{\prime}\right) & \longmapsto z^{\prime}
\end{aligned}
$$

is an increasing bijection (for the product order). Now, $\sigma_{[l, n]}$ is the longest element of $\mathfrak{S}_{[l, n]}$ and $\sigma_{[l+1, n]} \sigma_{[l, n]}=c_{[l, n-1]}$. Therefore, the map

$$
\begin{aligned}
{\left[1 ; c_{[l, n-1]}\right] } & \longrightarrow\left[\sigma_{[l+1, n]} ; \sigma_{[l, n]}\right] \\
z & \longmapsto \quad z \sigma_{[l, n]}
\end{aligned}
$$

is a decreasing bijection. So, if we denote by $\mathcal{P}(E)$ the set of subsets of a set $E$, then the maps

$$
\begin{aligned}
& \mathcal{P}([1, l-1]) \longrightarrow\left[1 ; c_{[1, l-1]}\right] \\
& I \longmapsto \\
& c_{I}
\end{aligned}
$$

and

$$
\begin{aligned}
\mathcal{P}([l, n-1]) & \longrightarrow\left[\sigma_{[l+1, n]} ; \sigma_{[l, n]}\right] \\
J & \longmapsto \quad c_{\bar{J}} \sigma_{[l, n]}
\end{aligned}
$$

are increasing bijections (here, $\bar{J}$ denotes the complement of $J$ ). On the other hand, the map

$$
\begin{aligned}
\mathcal{P}([1, l-1]) \times \mathcal{P}([l, n-1]) & \longrightarrow \mathcal{P}([1, n-1]) \\
(I, J) & \longmapsto \quad I \cup J
\end{aligned}
$$

is an increasing bijection. Finally, by composing all these bijections, we get an isomorphism of ordered sets

$$
\begin{aligned}
\alpha: \mathcal{P}([1, n-1]) & \longrightarrow \\
I & \longmapsto c_{I \cap[1, l-1]} c \bar{I}_{I \cap[l, n-1]} \sigma_{[l, n]} a_{l} .
\end{aligned}
$$

3.3 The elements $z \in[x ; y]$ such that $t z<z$

If $I \subseteq[1, n-1]$ is such that $t \alpha(I)<\alpha(I)$, we set $\tilde{\mu}_{I}=\tau_{A}\left(\mu_{\alpha(I), y}\right)$. So we can rephrase (?) as follows:

$$
\tilde{\mu}_{\varnothing} \neq 0 .
$$

But, by the induction formula that defines the $\mu$-polynomials and by 2.1 , we have, for all $I \subseteq[1, n-1]$ such that $t \alpha(I)<\alpha(I)$,

$$
\tilde{\mu}_{I}=1-\sum_{\substack{I \nsubseteq J \subseteq[1, n-1] \\ t \alpha(J)<\alpha(J)}} \tilde{\mu}_{J} .
$$


Let

$$
\mathcal{E}=\{I \in \mathcal{P}([1, n-1]) \mid t \alpha(I)<\alpha(I)\} .
$$

The set $\mathcal{E}$ is easy to describe:

Lemma 3.1 Let $I \subseteq[1, n-1]$. Then $t \alpha(I)>\alpha(I)$ if and only if $[1, l-1] \varsubsetneqq I$.

Proof of Lemma 3.1 By Lemma 1.1, we just need to show that

$$
\alpha(I)^{-1}(1)>0 \text { if and only if }[1, l-1] \varsubsetneqq I .
$$

For simplification, we set $A=[1, l-1] \cap I$ and $B=\overline{I \cap[l, n-1]}$. So $\alpha(I)=$ $c_{A} c_{B} \sigma_{[l, n]} a_{l}$.

First, assume that $[1, l-1] \nsubseteq I$. Then $0<c_{A}^{-1}(1)<n$, so $\sigma_{[l, n]}^{-1} c_{B}^{-1} c_{A}^{-1}(1)=$ $c_{A}^{-1}(1)$ and $\alpha(I)^{-1}(1)=a_{l}^{-1}\left(c_{A}^{-1}(1)\right)<0$ by 1.5 . This shows (\#) in this case.

Now, let us assume that $[1, l-1]=I$. Then $c_{A}=s_{1} \cdots s_{l-1}$ and $c_{B}=s_{l} \cdots s_{n-1}$ and so $c_{A}^{-1}(1)=l$ and $c_{B}^{-1}(l)=n$. In particular, $\alpha(I)^{-1}(1)=a_{l}^{-1} \sigma_{[l, n]}(n)=$ $a_{l}^{-1}(l)=-1<0$ by 1.5 . This shows (\#) again in this case.

Now, let us assume that $[1, l-1] \varsubsetneqq I$. Then $c_{A}^{-1}(1)=l$ and $c_{B}^{-1}(l)<n$ and so $\sigma_{[l, n]}^{-1} c_{B}^{-1} c_{A}^{-1}(1)>l$. So $\alpha(I)^{-1}(1)>0$ by 1.5 . The proof of (\#) is complete.

\subsection{Computation of the $\tilde{\mu}_{I}$ 's}

We shall now compute the family $\left(\tilde{\mu}_{I}\right)_{I \in \mathcal{E}}$ by descending induction on $|I|$, by using the formula 3.1. For this, the following well-known lemma will be useful.

Lemma 3.2 If $S$ is a finite set and $I \varsubsetneqq S$, then $\sum_{I \subseteq J \subseteq S}(-1)^{|J|}=0$.

To obtain the value of $\tilde{\mu}_{\varnothing}$, the proof goes in three steps.

$$
\text { If }[l, n-1] \subseteq I \varsubsetneqq[1, n-1] \text {, then } \tilde{\mu}_{I}=(-1)^{n-|I|} .
$$

Proof of 3.2 First, note that $I \in \mathcal{E}$ by Lemma 3.1. We argue by descending induction on $|I|$. If $|I|=n-2$, then $\tilde{\mu}_{I}=1$, as desired. Now, let us assume that $[l, n-1] \subseteq$ $I \varsubsetneqq[1, n-1]$ and that $\tilde{\mu}_{J}=(-1)^{n-|J|}$ for all $I \varsubsetneqq J \varsubsetneqq[1, n-1]$. Then, by 3.1, we have

$$
\tilde{\mu}_{I}=1-\sum_{I \nsubseteq J \varsubsetneqq[1, n-1]}(-1)^{n-|J|} .
$$

Therefore,

$$
\tilde{\mu}_{I}=1+(-1)^{n-|I|}+(-1)^{n-(n-1)}-\sum_{I \subseteq J \subseteq[1, n-1]}(-1)^{n-|J|}=(-1)^{n-|I|},
$$

the last equality following from Lemma 3.2. 
If $I \in \mathcal{E}$ is such that $[l, n-1] \nsubseteq I$ and $I \nsubseteq[1, l-1]$, then $\tilde{\mu}_{I}=0$.

Proof of 3.3 We shall again argue by descending induction on $|I|$. Let $I^{\prime}=I \cup[l, n-$ 1]. Then, by 3.1 , we have

$$
\tilde{\mu}_{I}=1-\sum_{\substack{J \in \mathcal{E} \\ I \nsubseteq J \text { and } I^{\prime} \subseteq J}} \tilde{\mu}_{J}-\sum_{\substack{J \in \mathcal{E} \\ I \nsubseteq J \text { and } I^{\prime} \nsubseteq J}} \tilde{\mu}_{J} .
$$

But, if $J \in \mathcal{E}$ is such that $I \varsubsetneqq J$ and $I^{\prime} \nsubseteq J$, (or, equivalently, $[l, n-1] \nsubseteq J$ ), then $\tilde{\mu}_{J}=0$ by the induction hypothesis. On the other hand, if $J \in \mathcal{E}$ is such that $I \varsubsetneqq J$ and $I^{\prime} \nsubseteq J$, then $\tilde{\mu}_{J}=(-1)^{n-|J|}$ by 3.2 . Therefore,

$$
\begin{aligned}
\tilde{\mu}_{I} & =1-\sum_{\substack{J \in \mathcal{E} \\
I \nsubseteq J \text { and } I^{\prime} \subseteq J}}(-1)^{n-|J|}=1-\sum_{I^{\prime} \subseteq J \subsetneq[1, n-1]}(-1)^{n-|J|} \\
& =-\sum_{I^{\prime} \subseteq J \subseteq[1, n-1]}(-1)^{n-|J|}=0
\end{aligned}
$$

by Lemma 3.2 .

$$
\text { If } I \subseteq[1, l-1] \text {, then } \tilde{\mu}_{I}=(-1)^{l-1-|I|} \text {. }
$$

Proof of 3.4 Note that $I \in \mathcal{E}$. We shall argue by descending induction on $|I|$. First, for all $J$ such that $I \varsubsetneqq J \subseteq[1, n-1]$, we have $t \alpha(I)>\alpha(I)$. Therefore, $\tilde{\mu}_{[1, l-1]}=1$, as desired.

Now, let $I \varsubsetneqq[1, l-1]$ and assume that, for all $I \varsubsetneqq J \subseteq[1, l-1]$, we have $\tilde{\mu}_{J}=$ $(-1)^{l-1-|J|}$. Then

$$
\tilde{\mu}_{I}=1-\sum_{\substack{J \in \mathcal{E} \\ I \nsubseteq J}} \tilde{\mu}_{I}
$$

Now, if $J \in \mathcal{E}$ is such that $I \varsubsetneqq J$, then three cases may occur:

- If $J \subseteq[1, l-1]$, then $\tilde{\mu}_{J}=(-1)^{l-1-|J|}$ by the induction hypothesis.

- If $J \nsubseteq[1, l-1]$ and $[l, n-1] \nsubseteq I$, then $\tilde{\mu}_{J}=0$ by 3.3 .

- If $[l, n-1] \subseteq J$, then $\tilde{\mu}_{J}=(-1)^{n-|J|}$.

Therefore, if we set $I^{\prime}=I \cap[l, n-1]$, then we get

$$
\tilde{\mu}_{I}=1-\sum_{I^{\prime} \subseteq J \varsubsetneqq[l, n-1]}(-1)^{n-|J|}-\sum_{I \nsubseteq J \subsetneq[1, l-1]}(-1)^{l-1-|J|} .
$$

But

$$
1-\sum_{I^{\prime} \subseteq J \subsetneq[l, n-1]}(-1)^{n-|J|}=-\sum_{I^{\prime} \subseteq J \subseteq[l, n-1]}(-1)^{n-|J|}=0
$$


and

$$
-\sum_{I \varsubsetneqq J \subsetneq[1, l-1]}(-1)^{l-1-|J|}=(-1)^{l-1-|I|}-\sum_{I \subseteq J \subsetneq[1, l-1]}(-1)^{l-1-|J|}=(-1)^{l-1-|I|}
$$

by Lemma 3.2. The proof is now complete.

As a special case of 3.4 , we get that

$$
\tilde{\mu}_{\varnothing}=(-1)^{l-1} .
$$

This shows (?). The proof of the Proposition 2.10 is complete.

\section{Consequences of Proposition 2.10}

The aim of this section is to prove the following

Proposition 4.1 Let $l \in\{0,1, \ldots, n\}$, let $\alpha, \beta \in Y_{l, n-l}$ and let $\sigma$ and $\sigma^{\prime} \in \mathfrak{S}_{l, n-l}$ be such that $\sigma \sim_{L} \sigma^{\prime}$. Assume that $b \geqslant(n-1) a$. Then

$$
\alpha a_{l} \sigma \beta^{-1} \sim_{L} a_{l} \sigma^{\prime} \beta^{-1}
$$

Remarks (1) The condition $\sigma \sim_{L} \sigma^{\prime}$ does not depend on the choice of $a$ and $b$ in $\Gamma$. Indeed, by [5, Theorem 1], $\sigma \sim_{L} \sigma^{\prime}$ in $W_{n}$ if and only if $\sigma \sim_{L} \sigma^{\prime}$ in $\mathfrak{S}_{l, n-l}$. But this last condition depends neither on the choice of $b$ (since $t \notin \mathfrak{S}_{l, n-l}$ ) nor on the choice of $a$ (provided that it is in $\Gamma_{>0}$ ).

(2) If $b>(n-1) a$, then the above proposition is proved in [4, Theorem 7.7] (see also [1, Corollary 5.2] for the exact bound) by a counting argument. The proof below will not use this counting argument but uses instead the proposition 2.10: it allows to extend the scope of validity to the case where $b=(n-1) a$ (this is compatible with $[2$, Conjecture A (b)]).

Proof First, recall that $a_{l} \sigma \beta^{-1} \sim_{L} a_{l} \sigma^{\prime} \beta^{-1}$ by Proposition 2.7. This shows that we may (and we will) assume that $\sigma=\sigma^{\prime}$. We want to show that $\alpha a_{l} \sigma \beta^{-1} \sim_{L} a_{l} \sigma \beta^{-1}$. We shall use induction on $n$. So let $\left(P_{n}\right)$ denote the following statement:

$\left(P_{n}\right)$ For all $l \in[0, n]$, for all sequences $1 \leqslant i_{1}<\cdots<i_{l} \leqslant n$, for all $\sigma \in \mathfrak{S}_{l, n-l}$ and for all $\beta \in Y_{l, n-l}$, we have $r_{i_{1}} r_{i_{2}} \cdots r_{i_{l}} \sigma \beta^{-1} \sim_{L} r_{1} r_{2} \cdots r_{l} \sigma \beta^{-1}$.

The property $\left(P_{1}\right)$ is vacuously true and the property $\left(P_{2}\right)$ can be easily checked by a straightforward computation. So we assume that $n \geqslant 3$ and $\left(P_{m}\right)$ holds for all $m<$ $n$. Now, let $l \in[0, n]$, let $1 \leqslant i_{1}<\cdots<i_{l} \leqslant n$ be a sequence of elements of $[1, n]$, let $\sigma \in \mathfrak{S}_{l, n-l}$ and let $\beta \in Y_{l, n-l}$. As a consequence of this induction hypothesis, we get:

Lemma 4.2 If $k \in[1, l]$ is such that $i_{k}<n$, then $r_{i_{1}} r_{i_{2}} \cdots r_{i_{l}} \sigma \beta^{-1} \sim_{L} r_{1} \cdots r_{k} r_{i_{k+1}}$ $\cdots r_{i_{l}} \sigma \beta^{-1}$. 
Proof Let $w=r_{i_{1}} r_{i_{2}} \cdots r_{i_{l}} \sigma \beta^{-1}$ and $w^{\prime}=r_{1} \cdots r_{k} r_{i_{k+1}} \cdots r_{i_{l}} \sigma \beta^{-1}$. Let us write $w=$ $v x^{-1}$ and $w^{\prime}=v^{\prime} x^{\prime-1}$ with $v, v^{\prime} \in W_{i_{k}}$ and $x, x^{\prime} \in X_{n}^{\left(i_{k}\right)}$. First, note that

$$
w w^{\prime-1}=\left(r_{i_{1}} \cdots r_{i_{k}}\right) \cdot\left(r_{1} \cdots r_{k}\right)^{-1} \in W_{i_{k}}
$$

Therefore, $x=x^{\prime}$ and

$$
v v^{\prime-1}=\left(r_{i_{1}} \cdots r_{i_{k}}\right) \cdot\left(r_{1} \cdots r_{k}\right)^{-1} \in W_{i_{k}} .
$$

Moreover, by Lemma 1.4 , we have $0<x(1)<\cdots<x\left(i_{k}\right)$. So, if $i \in\left[1, i_{k}\right]$, then $v^{-1}(i)<0$ (resp. $v^{\prime-1}(i)<0$ ) if and only if $i \in\left\{i_{1}, \ldots, i_{k}\right\}$ (resp. $\{1, \ldots, k\}$ ). So, by Lemma 1.2, we have

$$
v=r_{i_{1}} \cdots r_{i_{k}} \tau \gamma^{-1} \text { and } v^{\prime}=r_{1} \cdots r_{k} \tau \gamma^{-1}
$$

where $\tau \in \mathfrak{S}_{k, i_{k}-k}$ and $\gamma \in Y_{k, i_{k}-k}$. But, since $i_{k}<n$, it follows from the induction hypothesis that $v \sim_{L} v^{\prime}$. Here, note that $v \sim_{L} v^{\prime}$ in $W_{i_{k}}$ if and only if $v \sim_{L} v^{\prime}$ in $W_{n}$ (see [5, Theorem 1]). So, by [11, Proposition 9.11], we get that $w \sim_{L} w^{\prime}$.

\section{Corollary 4.3}

(a) If $i_{l}<n$, then $r_{i_{1}} r_{i_{2}} \cdots r_{i_{l}} \sigma \beta^{-1} \sim_{L} r_{1} r_{2} \cdots r_{l} \sigma \beta^{-1}$.

(b) If $i_{l}=n$, then $r_{i_{1}} r_{i_{2}} \cdots r_{i_{l}} \sigma \beta^{-1} \sim_{L} r_{1} r_{2} \cdots r_{l-1} r_{n} \sigma \beta^{-1}$.

By Corollary 4.3, we only need to show that

$$
r_{1} r_{2} \cdots r_{l-1} r_{n} \sigma \beta^{-1} \sim_{L} r_{1} r_{2} \cdots r_{l} \sigma \beta^{-1}
$$

Now, let us write $\sigma=(\lambda, \mu)$, where $\lambda \in \mathfrak{S}_{[1, l]}$ and $\mu \in \mathfrak{S}_{[l+1, n]}$. Three cases may occur:

- Case 1: If $\lambda=1$ and $\mu=\sigma_{[l+1, n]}$, then $\sigma=\sigma_{[l+1, n]}$. Since $r_{1} r_{2} \cdots r_{l-1} r_{k} \sigma \beta^{-1}=$ $s_{k} s_{k-1} \cdots s_{l+1} \sigma \beta^{-1}$ for all $k>l$, we have

$$
\begin{aligned}
r_{1} \cdots r_{l-1} r_{n} \sigma \beta^{-1} & \leqslant_{L} r_{1} \cdots r_{l-1} r_{n-1} \sigma \beta^{-1} \leqslant_{L} \cdots \\
& \leqslant_{L} r_{1} \cdots r_{l-1} r_{l+1} \sigma \beta^{-1} \leqslant_{L} r_{1} \cdots r_{l-1} r_{l} \sigma \beta^{-1} .
\end{aligned}
$$

On the other hand, by Proposition 2.10 and Lemma 2.6, we get $r_{1} \cdots r_{l-1} r_{l} \sigma \beta^{-1} \leqslant L$ $r_{1} \cdots r_{l-1} r_{n} \sigma \beta^{-1}$. This shows (?) in this particular case.

- Case 2: If $\mu \neq \sigma_{[l+1, n]}$, then $n \geqslant l+2$ and there exists $k \in[l+1, n-1]$ such that $s_{k} \sigma>\sigma$. Let $i$ be maximal such that $s_{i} \sigma>\sigma$. We shall prove (?) by descending induction on $i$. For simplification, let $x=r_{1} \cdots r_{l-1} r_{n} \sigma \beta^{-1}$.

First, if $i=n-1$, then, by 1.3 , we have (since $n-2>l-1$ )

$$
\begin{gathered}
s_{n-2} x=r_{1} \cdots r_{l-1} s_{n-2} r_{n} \sigma \beta^{-1}=r_{1} \cdots r_{l-1} r_{n} s_{n-1} \sigma \beta^{-1}>s_{n-2} x, \\
s_{n-1} x=r_{1} \cdots r_{l-1} s_{n-1} r_{n} \sigma \beta^{-1} r_{1} \cdots r_{l-1} r_{n-1} \sigma \beta^{-1}<x
\end{gathered}
$$


and

$$
s_{n-2} s_{n-1} x=r_{1} \cdots r_{l-1} s_{n-2} r_{n-1} \sigma \beta^{-1}=r_{1} \cdots r_{l-1} r_{n-2} \sigma \beta^{-1}<s_{n-1} x .
$$

So $x \sim_{L} s_{n-1} x$ by Corollary 2.2. On the other hand, by Corollary 4.3, we have $s_{n-1} x \sim_{L} r_{1} \cdots r_{l} \sigma \beta^{-1}$, so we get (?) in this case.

Now, assume that $l+1 \leqslant i<n-1$. Then $s_{i+1} \sigma<\sigma$ (by the maximality of $i$ ). Two cases may occur:

Subcase 1: If $s_{i} s_{i+1} \sigma<s_{i+1} \sigma$, then we set $\tau=s_{i+1} \sigma<\sigma$ and $y=r_{1} \cdots r_{l-1} r_{n}$. $\tau \beta^{-1}$. Then $y=s_{i} x<x$ by 1.3 . Moreover, still by 1.3 , we have

$$
s_{i-1} x=r_{1} \cdots r_{l-1} r_{n} s_{i} \sigma \beta^{-1}>x
$$

and

$$
s_{i-1} s_{i} x=r_{1} \cdots r_{l-1} r_{n} s_{i} s_{i+1} \sigma \beta^{-1}<s_{i} x .
$$

So $x \sim_{L} y$ by Corollary 2.2. But, by the induction hypothesis (and since $s_{i+1} \tau>\tau$ ), we have $y \sim_{L} a_{l} \tau \beta^{-1}$. But $\sigma \sim_{L} \tau$ (again by Corollary 2.2 and since $s_{i} \tau<\tau<\sigma=$ $\left.s_{i+1} \tau<s_{i} \sigma\right)$, so $a_{l} \sigma \beta^{-1} \sim_{L} a_{l} \tau \beta^{-1}$ by (*). This shows (?).

Subcase 2: If $s_{i} s_{i+1} \sigma>s_{i+1} \sigma$, then $s_{i+1} s_{i} \sigma>s_{i} \sigma$ (by an easy application of Lemma 1.1) so, if we set $\tau=s_{i} \sigma$ and $y=r_{1} \cdots r_{l-1} r_{n} \tau \beta^{-1}$, we have, by the induction hypothesis, $y \sim_{L} a_{l} \tau \beta^{-1}$. Moreover, $s_{i+1} \tau>\tau=s_{i} \sigma>\sigma>s_{i+1} \sigma$ and, by the same argument as in the subcase 1 , we have $s_{i} y>y=s_{i-1} x>x>s_{i} x$. So $x \sim_{L} y$, $\sigma \sim_{L} \tau$. So it follows from (*) and $x \sim_{L} a_{l} \sigma \beta^{-1}$, as required.

Case 3: If $\lambda \neq 1$, then we set $x=r_{1} \cdots r_{l-1} r_{n} \sigma \beta^{-1}$ and $y=r_{1} \cdots r_{l} \sigma \beta^{-1}$. We want to show that $x \sim_{L} y$. For this, let $x^{\prime}=w_{n} x, y^{\prime}=w_{n} y, \sigma^{\prime}=\sigma_{n} \sigma \sigma_{n}^{-1} \sigma_{n-l, l}$ and $\beta^{\prime}=$ $\beta \sigma_{n} \sigma_{n-l, l}$. Then, by Proposition 1.3,

$$
x^{\prime}=r_{l} r_{l+1} \cdots r_{n-1} \sigma^{\prime} \beta^{\prime-1} \quad \text { and } \quad y^{\prime}=r_{l} r_{l+1} \cdots r_{n-2} r_{n} \sigma^{\prime} \beta^{\prime-1} .
$$

But, by Corollary 4.3, we have

$$
x^{\prime} \sim_{L} r_{1} \cdots r_{n-l} \sigma^{\prime} \beta^{\prime-1} \text { and } y^{\prime} \sim_{L} r_{1} \cdots r_{n-l-1} r_{n} \sigma^{\prime} \beta^{\prime-1} .
$$

Now, if we write $\sigma^{\prime}=\left(\lambda^{\prime}, \mu^{\prime}\right)$, with $\lambda^{\prime} \in \mathfrak{S}_{[1, n-l]}$ and $\mu^{\prime} \in \mathfrak{S}_{[n-l+1, n]}$, we have $\mu^{\prime} \neq$ $\sigma_{[n-l+1, n]}$ (because $\lambda \neq 1$ ). So, by Case 2 , we have

$$
r_{1} \cdots r_{n-l-1} r_{n} \sigma^{\prime} \beta^{\prime-1} \sim_{L} r_{1} \cdots r_{n-l} \sigma^{\prime} \beta^{\prime-1} .
$$

Therefore, $x^{\prime}=w_{n} x \sim_{L} y^{\prime}=w_{n} y$, and so $x \sim_{L} y$ by [11, Corollary 11.7].

Corollary 4.4 Let $l \in\{1, \ldots, n\}$, let $1 \leqslant i_{1}<\cdots<i_{l} \leqslant n$, let $\sigma \in \mathfrak{S}_{n}$, let $\beta \in Y_{l, n-l}$ and let $k \in[1, l]$ be such that $b \geqslant\left(i_{k}-1\right) a$. Then

$$
r_{i_{1}} \cdots r_{i_{l}} \sigma \sim_{L} r_{1} \cdots r_{k} r_{i_{k+1}} \cdots r_{i_{l}} \sigma
$$


Proof The proof proceeds essentially as in Lemma 4.2. Let $w=r_{i_{1}} \cdots r_{i_{l}} \sigma$, let $w^{\prime}=$ $r_{1} \cdots r_{k} r_{i_{k+1}} \cdots r_{i_{l}} \sigma$ and let us write $w=v x^{-1}$ and $w^{\prime}=v^{\prime} x^{\prime-1}$ with $v, v^{\prime} \in W_{i_{k}}$ and $x, x^{\prime} \in X_{n}^{\left(i_{k}\right)}$.

Since $w^{\prime} w^{-1}=\left(r_{1} \cdots r_{k}\right)^{-1}\left(r_{i_{1}} \cdots r_{i_{k}}\right) \in W_{i_{k}}$, we get that $x=x^{\prime}$. The same argument as in Lemma 4.2 shows that $v=r_{i_{1}} \cdots r_{i_{k}} \tau$ and $v^{\prime}=r_{1} \cdots r_{k} \tau^{\prime}$ for some $\tau$, $\tau^{\prime} \in \mathfrak{S}_{i_{k}}$. But $v^{\prime} v^{-1}=w^{\prime} w^{-1}=\left(r_{1} \cdots r_{k}\right)^{-1}\left(r_{i_{1}} \cdots r_{i_{k}}\right)$, so $\tau=\tau^{\prime}$. Now, by Proposition 4.1, $v \sim_{L} v^{\prime}$. So $w \sim_{L} w^{\prime}$ by [11, Proposition 9.11].

\section{Proof of Proposition 2.11}

Notation. In this section, and only in this section, we assume that $1 \leqslant l \leqslant n-1$ and that $(n-2) a<b \leqslant(n-1) a$.

We define a sequence $\left(C_{j}\right)_{l-1 \leqslant j \leqslant n-1}$ by induction as follows:

$$
\left\{\begin{array}{l}
C_{l-1}=1, \\
C_{l}=C_{s_{l}}, \\
C_{j+1}=C_{s_{j+1}} C_{j}-C_{j-1}, \quad \text { if } l \leqslant j \leqslant n-2 .
\end{array}\right.
$$

Let $\mu$ denote the coefficient of $C_{a_{l-1} \sigma_{[l, n]}}$ in the expansion of $C_{n-1} C_{a_{l} \sigma_{[l, n]}}$ in the Kazhdan-Lusztig basis. To prove Proposition 2.11, it is sufficient to show the following statement:

$$
\mu= \begin{cases}1 & \text { if } b=(n-1) a \\ Q^{-1} q^{n-1}+Q q^{1-n} & \text { if }(n-2) a<b<(n-1) a .\end{cases}
$$

Proof of 5.1 If $r \in \mathbb{Z}$, we set

$$
\mathcal{H}_{n}[r]=\underset{\ell_{t}(w) \leqslant r}{\oplus} A T_{w}=\underset{\ell_{t}(w) \leqslant r}{\oplus} A C_{w}
$$

We shall show that

$$
\begin{aligned}
C_{n-1} C_{a_{l} \sigma_{[l, n]}} \equiv & T_{s_{n-1} \cdots s_{l+1} s_{l} a_{l} \sigma_{[l, n]}} \\
& +Q^{-1} q^{n-1} T_{a_{l-1} \sigma_{[l, n]}} \bmod \left(\mathcal{H}_{n}[l-2]+\mathcal{H}_{n}^{<0}\right) .
\end{aligned}
$$

The statement 5.2 will be proved at the end of this section. Let us conclude the proof of 5.1, assuming that 5.2 holds.

Let

$$
\tilde{\mu}= \begin{cases}1 & \text { if } b=(n-1) a, \\ Q^{-1} q^{n-1}+Q q^{1-n} & \text { if }(n-2) a<b<(n-1) a .\end{cases}
$$

We want to show that $\mu=\tilde{\mu}$. But, by 5.2 , we have

$$
\begin{aligned}
& C_{n-1} C_{a_{l} \sigma_{[l, n]}}-C_{s_{n-1} \cdots s_{l+1} s_{l} a_{l} \sigma_{[l, n]}}-\tilde{\mu} C_{a_{l-1} \sigma_{[l, n]}} \\
& \in \mathcal{H}_{n}[l-2]+\mathcal{H}_{n}^{<0}+\underset{w<a_{l-1} \sigma_{[l+1, n]}}{\oplus} A T_{w} .
\end{aligned}
$$


Since

$$
\underset{w<a_{l-1} \sigma_{[l+1, n]}}{\bigoplus} A T_{w}=\underset{w<a_{l-1} \sigma_{[l+1, n]}}{\bigoplus} A C_{w},
$$

there exists a family $\left(v_{w}\right) \ell_{t}(w) \leqslant l-2$ or $w<a_{l-1} \sigma_{[l+1, n]}$ of elements of $A \geqslant 0$ such that

$$
C_{n-1} C_{a_{l} \sigma_{[l, n]}}-C_{S_{n-1} \cdots s_{l+1} s_{l} a_{l} \sigma_{[l, n]}}-\tilde{\mu} C_{a_{l-1} \sigma_{[l, n]}}-\sum_{\begin{array}{c}
\ell_{t}(w) \leqslant l-2 \\
\text { or } a_{l-1} \sigma_{[l+1, n]}
\end{array}} v_{w} C_{w} \in \mathcal{H}_{n}^{<0} .
$$

Let $v_{w}^{\prime}=v_{w}+\bar{v}_{w}-\tau_{A}\left(v_{w}\right)$. Then

$$
C_{n-1} C_{a_{l} \sigma_{[l, n]}}-C_{s_{n-1} \cdots s_{l+1} s_{l} a_{l} \sigma_{[l, n]}}-\tilde{\mu} C_{a_{l-1} \sigma_{[l, n]}}-\sum_{\begin{array}{c}
\ell_{t}(w) \leqslant l-2 \\
\text { or } a_{l-1} \sigma_{[l+1, n]}
\end{array}} v_{w}^{\prime} C_{w} \in \mathcal{H}_{n}^{<0}
$$

and $\bar{v}_{w}^{\prime}=v_{w}$. So, if we set

$$
C=C_{n-1} C_{a_{l} \sigma_{[l, n]}}-C_{s_{n-1} \cdots s_{l+1} s_{l} a_{l} \sigma_{[l, n]}}-\tilde{\mu} C_{a_{l-1} \sigma_{[l, n]}}-\sum_{\substack{\ell_{t}(w) \leqslant l-2 \\ \text { or } a_{l-1} \sigma_{[l+1, n]}}} v_{w}^{\prime} C_{w},
$$

then

$$
\bar{C}=C \quad \text { and } \quad C \in \mathcal{H}_{n}^{<0} .
$$

So $C=0$ by [11, Theorem 5.2], and so $\mu=\tilde{\mu}$, as expected.

So it remains to prove the statement 5.2:

Proof of 5.2 First of all, we have $C_{a_{l} \sigma_{[l, n]}}=C_{a_{l}} C_{\sigma_{[l, n]}}$, since the supports of $a_{l}$ and $\sigma_{[l, n]}\left(\right.$ in $S_{n}$ ) are disjoint. Moreover, since $l \leqslant n-1$ (i.e. $\left.a_{l} \in W_{n-1}\right)$ and $b>(n-2) a$, it follows from [1, Propositions 2.5 and 5.1] that

$$
C_{a_{l}}=\left(T_{t_{1}}+Q^{-1}\right)\left(T_{t_{2}}+Q^{-1}\right) \cdots\left(T_{t_{l}}+Q^{-1}\right) T_{\sigma_{l}}^{-1} .
$$

Let $\mathcal{H}\left(\mathfrak{S}_{n}\right)$ denote the sub- $A$-algebra of $\mathcal{H}_{n}$ generated by $T_{S_{1}}, \ldots, T_{S_{n-1}}$. It is the Hecke algebra of $\mathfrak{S}_{n}$ (with parameter $a$ ). Then $\mathcal{H}_{n}[l-2]$ is a sub- $A$-module of $\mathcal{H}_{n}$. Therefore, it follows from 5.3 that

$$
C_{a_{l}} \equiv\left(T_{w_{l}}+Q^{-1} \sum_{1 \leqslant i \leqslant l} T_{t_{1} \cdots t_{i-1} t_{i+1} \cdots t_{l}}\right) T_{\sigma_{l}}^{-1} \quad \bmod \mathcal{H}_{n}[l-2]
$$

But, if $1 \leqslant i \leqslant l$, then

$$
t_{1} \cdots t_{i-1} t_{i+1} \cdots t_{l}=s_{i} s_{i+1} \cdots s_{l-1} a_{l-1} \sigma_{l-1} s_{l-1} \cdots s_{i+1} s_{i}
$$

and $\sigma_{l}=s_{l+1-i} \cdots s_{l-2} s_{l-1} \sigma_{l-1} s_{l-1} \cdots s_{i+1} s_{i}$. Moreover,

$$
\ell\left(\sigma_{l}\right)=\ell\left(s_{l+1-i} \cdots s_{l-2} s_{l-1}\right)+\ell\left(\sigma_{l-1} s_{l-1} \cdots s_{i+1} s_{i}\right) .
$$


Therefore,

$$
C_{a_{l}} \equiv T_{a_{l}}+Q^{-1} \sum_{1 \leqslant i \leqslant l} T_{s_{i} s_{i+1} \cdots s_{l-1}} T_{a_{l-1}}\left(T_{s_{l+1-i} \cdots s_{l-2} s_{l-1}}\right)^{-1} \bmod \mathcal{H}_{n}[l-2] .
$$

Finally, we get

$$
C_{a_{l} \sigma_{[l, n]}} \equiv T_{a_{l}} C_{\sigma_{[l, n]}}+Q^{-1} \sum_{1 \leqslant i \leqslant l} T_{c_{[i, l-1]}} T_{a_{l-1}}\left(T_{c_{[l+1-i, l-1]}}\right)^{-1} C_{\sigma_{[l, n]}} \bmod \mathcal{H}_{n}[l-2] .
$$

Now, if $l-1 \leqslant j \leqslant n-1$, then

$$
\begin{aligned}
C_{j} C_{a_{l} \sigma_{[l, n]}} \equiv & \sum_{i=l-1}^{j} q^{i-j} T_{d_{[l, i]}} T_{a_{l}} C_{\sigma_{[l, n]}} \\
& +Q^{-1} C_{j} \sum_{1 \leqslant i \leqslant l} T_{c_{[i, l-1]}} T_{a_{l-1}}\left(T_{c_{[l+1-i, l-1]}}\right)^{-1} C_{\sigma_{[l, n]}} \bmod \mathcal{H}_{n}[l-2] .
\end{aligned}
$$

Proof of 5.4 We shall argue by induction on $j$. The cases where $j=l-1$ or $j=l$ are obvious. So assume that $j \in[l, n-2]$ and that 5.4 holds for $j$. By the induction hypothesis, we get

$$
\begin{aligned}
C_{j+1} C_{a_{l} \sigma_{[l, n]}} \equiv & C_{s_{j+1}} \sum_{i=l-1}^{j} q^{i-j} T_{d_{[l, i]}} T_{a_{l}} C_{\sigma_{[l, n]}}-\sum_{i=l-1}^{j-1} q^{i-j+1} T_{d_{[l, i]}} T_{a_{l}} C_{\sigma_{[l, n]}} \\
& +Q^{-1} C_{j+1} \sum_{i \leqslant l} T_{c_{[i, l-1]}} T_{a_{l-1}}\left(T_{c_{[l+1-i, l-1]}}\right)^{-1} C_{\sigma_{[l, n]}} \\
& \bmod \mathcal{H}_{n}[l-2] .
\end{aligned}
$$

Now,

$$
C_{s_{j+1}} T_{d_{[l, j]}} T_{a_{l}} C_{\sigma_{[l, n]}}=T_{d_{[l, j+1]}} T_{a_{l}} C_{\sigma_{[l, n]}}+q^{-1} T_{d_{[l, j]}} T_{a_{l}} C_{\sigma_{[l, n]}}
$$

and, if $l-1 \leqslant i<j$, then

$$
C_{s_{j+1}} T_{d_{[l, i]}} T_{a_{l}} C_{\sigma_{[l, n]}}=T_{d_{[l, i]}} T_{a_{l}} C_{s_{j+1}} C_{\sigma_{[l, n]}}=\left(q+q^{-1}\right) T_{d_{[l, i]}} T_{a_{l}} C_{\sigma_{[l, n]}} .
$$

Now 5.4 follows from a straightforward computation.

Since $d_{[l, i]} \in Y_{l, n-l}$, we have

$$
T_{d_{[l, i]}} T_{a_{l}} C_{\sigma_{[l, n]}}=T_{d_{[l, i]} a_{l}} C_{\sigma_{[l, n]}} \equiv T_{d_{[l, i]} a_{l} \sigma_{[l, n]}} \quad \bmod \mathcal{H}_{n}^{<0}
$$

so, by 5.4 , we get

$$
\begin{aligned}
C_{n-1} C_{a_{l} \sigma_{[l, n]}} \equiv & T_{d_{[l, n-1]} a_{l} \sigma_{[l, n]}} \\
& +Q^{-1} C_{n-1} \sum_{1 \leqslant i \leqslant l} T_{c_{[i, l-1]}} T_{a_{l-1}}\left(T_{c_{[l+1-i, l-1]}}\right)^{-1} C_{\sigma_{[l, n]}} \\
& \bmod \left(\mathcal{H}_{n}[l-2]+\mathcal{H}_{n}^{<0}\right) .
\end{aligned}
$$


For $1 \leqslant i \leqslant l$, let $\mathcal{X}_{i}=Q^{-1} C_{n-1} T_{c_{[i, l-1]}} T_{a_{l-1}}\left(T_{c_{[l+1-i, l-1]}}\right)^{-1} C_{\sigma_{[l, n]}}$. There exists a family $\left(f_{I}\right)_{I \subseteq[l, n-1]}$ of elements of $\mathbb{Z}$ such that $C_{n-1}=\sum_{I \subseteq[l, n-1]} f_{I} C_{d_{I}}$. Moreover, $f_{[l, n-1]}=1$. Also,

$$
\left(T_{c_{[l+1-i, l-1]}}\right)^{-1}=\sum_{J \subseteq[l+1-i, l-1]}\left(q-q^{-1}\right)^{i-1-|J|} T_{c_{J}}
$$

Therefore,

$$
\mathcal{X}_{i}=\sum_{\substack{I \subseteq[l, n-1] \\ J \subseteq[l+1-i, l-1]}} f_{I} Q^{-1}\left(q-q^{-1}\right)^{i-1-|J|} C_{d_{I}} T_{c_{[i, l-1]}} T_{a_{l-1}} T_{c_{J}} C_{\sigma_{[l, n]}}
$$

Let $\Delta_{i, I, J}=f_{I} Q^{-1}\left(q-q^{-1}\right)^{i-1-|J|} C_{d_{I}} T_{c_{[i, l-1]}} T_{a_{l-1}} T_{c_{J}} C_{\sigma_{[l, n]}}$. If we express $\Delta_{i, I, J}$ in the standard basis $\left(T_{w}\right)_{w \in W_{n}}$, then the degrees of the coefficients are bounded by $-b+(i-1-|J|+|I|) a$. Since $b>(n-2) a$, this degree is in $\Gamma_{<0}$, except if $i=l$, $J=\varnothing$ and $I=[l, n-1]$. Therefore,

$$
C_{n-1} C_{a_{l} \sigma_{[l, n]}} \equiv T_{d_{[l, n-1]} a_{l} \sigma_{[l, n]}}+\Delta_{l,[l, n-1], \varnothing} \bmod \left(\mathcal{H}_{n}[l-2]+\mathcal{H}_{n}^{<0}\right) .
$$

But

$$
\begin{aligned}
\Delta_{l,[l, n-1], \varnothing} & =Q^{-1}\left(q-q^{-1}\right)^{l-1} C_{s_{n-1} \cdots s_{l}} T_{a_{l-1}} C_{\sigma_{[l, n]}} \\
& =Q^{-1}\left(q-q^{-1}\right)^{l-1} T_{a_{l-1}} C_{s_{n-1} \cdots s_{l}} C_{\sigma_{[l, n]}} \\
& =Q^{-1}\left(q-q^{-1}\right)^{l-1}\left(q+q^{-1}\right)^{n-l} T_{a_{l-1}} C_{\sigma_{[l, n]}},
\end{aligned}
$$

the last equality following from Theorem 2.1 (a). So $\Delta_{l,[l, n-1], \varnothing} \equiv Q^{-1} q^{n-1} T_{a_{l-1}}$. $C_{\sigma_{[l, n]}} \bmod \mathcal{H}_{n}^{<0}$. The proof of 5.2 is complete.

\section{Consequences of Proposition 2.11}

The aim of this section is to prove the following proposition:

Proposition 6.1 Let $l \in\{1, \ldots, n\}$ and assume that $b \leqslant(n-1) a$. Then

$$
s_{1} s_{2} \cdots s_{n-1} a_{l-1} \sigma_{[l, n-1]} \sim_{L} t s_{1} s_{2} \cdots s_{n-1} a_{l-1} \sigma_{[l, n-1]} .
$$

Proof Let $u_{l, n}=t s_{1} s_{2} \cdots s_{n-1} a_{l-1} \sigma_{[l, n-1]}=t s_{1} \cdots s_{l-1} a_{l-1} s_{l} \cdots s_{n-1} \sigma_{[l, n-1]}=$ $a_{l} \sigma_{[l, n]}$. We need to show that $t u_{l, n} \sim_{L} u_{l, n}$ (note that $t u_{l, n} \leqslant u_{l, n}$ ). We shall argue by induction on $n$, the cases where $n=1$ or 2 being obvious. So assume that $n \geqslant 3$ and that $t u_{l, n-1} \sim_{L} u_{l, n-1}$ if $b \leqslant(n-2) a$.

First, assume that $b \leqslant(n-2) a$. Then

$$
\begin{cases}u_{l, n}=u_{l, n-1} s_{n-1} \cdots s_{l+1} s_{l} & \text { if } l \leqslant n-1 \\ u_{l, n}=a_{n}=u_{l-1, n-1} s_{n-1} \cdots s_{2} s_{1} t & \text { if } l=n\end{cases}
$$


By the induction hypothesis, we have $t u_{k, n-1} \sim_{L} u_{k, n-1}$ so, since $s_{n-1} \cdots s_{l+1} s_{l}$ and $s_{n-1} \cdots s_{2} s_{1} t$ belong to $X_{n}^{-1}$, it follows from [11, Proposition 9.11] that $t u_{l, n} \sim_{L} u_{l, n}$.

This means that we may, and we will, assume that $(n-2) a<b \leqslant(n-1) a$. But, by Proposition 2.11, we have $a_{l-1} \sigma_{[l, n]} \leqslant{ }_{L} a_{l} \sigma_{[l, n]}=u_{l, n}$. On the other hand,

$$
\begin{aligned}
t u_{l, n} & =c_{[1, l-1]} a_{l-1} \sigma_{[l, n]} \leqslant L c_{[2, l-1]} a_{l-1} \sigma_{[l, n]} \leqslant L \\
& \leqslant L s_{l-1} a_{l-1} \sigma_{[l, n]} \leqslant L a_{l-1} \sigma_{[l, n]} .
\end{aligned}
$$

So $t u_{l, n} \sim_{L} u_{l, n}$, as desired.

Remark 6.2 Note that the converse of Proposition 6.1 also holds. Indeed, if $b>(n-$ 1) $a$ and if $x \sim_{L} y$ for some $x$ and $y$ in $W_{n}$, then $\ell_{t}(x)=\ell_{t}(y)$ (see [4, Theorem 7.7] and [1, Corollary 5.2]).

Corollary 6.2 Let $l \in\{1,2, \ldots, n\}$ and let $\beta \in Y_{l-1, n-l}$. Then

$$
s_{1} s_{2} \cdots s_{n-1} a_{l-1} \sigma_{[l, n-1]} \beta^{-1} \sim_{L} t s_{1} s_{2} \cdots s_{n-1} a_{l-1} \sigma_{[l, n-1]} \beta^{-1} .
$$

Proof Let $w=s_{1} s_{2} \cdots s_{n-1} a_{l} \sigma_{[l+1, n-1]} \beta^{-1}$. We want to show that $w \sim_{L} t w$. We shall argue by induction on $\ell(\beta)$. If $\ell(\beta)=0$ (i.e. $\beta=1$ ), this is just the proposition 6.1. Sp we assume now that $\ell(\beta) \geqslant 1$. We shall use the $*$-operation (see $\S 2.3$ ). For this, we need to study the action of the $\gamma_{i}$ 's on $w$, when possible.

We have $\sigma_{[l, n-1]} a_{l-1}=a_{l-1} \sigma_{[l, n-1]}$, so

$$
w=s_{1} s_{2} \cdots s_{n-1} \sigma_{[l, n-1]}\left(a_{l-1} \beta\right)^{-1}=s_{1} s_{2} \cdots s_{l-1} \sigma_{[l, n]}\left(a_{l-1} \beta\right)^{-1} .
$$

Let $1 \leqslant j_{1}<\cdots<j_{l-1} \leqslant n-1$ be the unique sequence such that $a_{l} \beta=r_{j_{1}} r_{j_{2}} \ldots$ $r_{j_{l-1}}$. Since $\ell(\beta)>0$, we have $\left(j_{1}, j_{2}, \ldots, j_{l-1}\right) \neq(1,2, \ldots, l-1)$, so there exists $k \in[1, l-1]$ such that $j_{k}-j_{k-1} \geqslant 2$ (where $j_{0}=0$ by convention). Note that $j_{k}<n$ so $j_{k}+1 \in[2, n]$. We have, by 1.14

$$
\begin{aligned}
w\left(j_{k}\right) s_{1} \cdots s_{l-1} \sigma_{[l, n]}\left(r_{j_{1}} \cdots r_{j_{l-1}}\right)^{-1}\left(j_{k}\right) & =s_{1} \cdots s_{l-1} \sigma_{[l, n]}(k-l) \\
& =-s_{1} \cdots s_{l-1}(l-k) \\
& =-(l+1-k)<0
\end{aligned}
$$

and

$$
\begin{aligned}
w\left(j_{k}-1\right) s_{1} \cdots s_{l-1} \sigma_{[l, n]}\left(r_{j_{1}} \cdots r_{j_{l-1}}\right)^{-1}\left(j_{k}-1\right) & =s_{1} \cdots s_{l-1} \sigma_{[l, n]}(l+q) \\
& =s_{1} \cdots s_{l-1}(n+1-q) \\
& =n+1-q>0
\end{aligned}
$$

for some $q \in[1, n+1-l]$. Moreover, a similar computation shows that (with the convention that $j_{l}=n+1$ )

$$
w\left(j_{k}\right)= \begin{cases}-(l-k) & \text { if } j_{k+1}=j_{k}+1 \\ n-q & \text { if } j_{k+1} \geqslant j_{k}+2\end{cases}
$$


In any case, we have

$$
w\left(j_{k}\right)<w\left(j_{k}+1\right)<w\left(j_{k}-1\right)
$$

This shows that

$$
w s_{j_{k}-1} s_{j_{k}}<w s_{j_{k}-1}<w<w s_{j_{k}},
$$

So $w \in \mathcal{D}_{j_{k}-1}\left(W_{n}\right)$ and $\gamma_{j_{k}-1}(w)=w s_{j_{k}-1}<w$. Now, let $\beta^{\prime}=s_{j_{k}} \beta$. An easy computation as above shows that $\beta^{\prime}<\beta$, so that $\beta^{\prime} \in Y_{l, n-1-l}$ by Deodhar's Lemma (see [7, Lemma 2.1.2]). So $\gamma_{j_{k}}(w)=s_{1} \cdots s_{n-1} a_{l-1} \sigma_{[l, n-1]} \beta^{\prime-1}$ where $\beta^{\prime} \in Y_{l, n-1-l}$ is such that $\ell\left(\beta^{\prime}\right)=\ell(\beta)-1$. But, by Corollary 2.9, we have $t \gamma_{i}(w)=\gamma_{i}(t w)$. So, by 2.4 and by the induction hypothesis, we get that $w \sim_{L} t w$, as desired.

\section{Proof of Theorem 1.5}

\subsection{Knuth Relations}

By recent results of Taskin [14, Theorems 1.2 and 1.3], the equivalence relations $\sim_{R}^{r}$ and $\simeq_{R}^{r}$ can be described using generalisations of Knuth relations (for the relation $\simeq_{R}^{r}$, a similar result has been obtained independently by Pietraho [12, Theorems 3.8 and 3.9] using other kinds of Knuth relations). We shall recall here Taskin's construction. For this, we shall need the following notation: if $0 \leqslant r \leqslant n-2$, we denote by $\mathcal{E}_{n}^{(r)}$ the set of elements $w \in W_{n}$ such that $|w(1)|>|w(i)|$ for $i \in\{2,3, \ldots, r+2\}$ and such that the sequence $(w(2), w(3), \ldots, w(r+2))$ is a shuffle of a positive decreasing sequence and a negative increasing sequence. If $r \geqslant n-1$, we set $\mathcal{E}_{n}^{(r)}=\varnothing$. Following [14, Definition 1.1], we introduce three relations which will be used to generate the relations $\sim_{R}^{r}$ and $\simeq_{R}^{r}$.

Let $w, w^{\prime} \in W_{n}$ and let $r \geqslant 0$ :

- We write $w \smile_{1} w^{\prime}$ if there exists $i \geqslant 2$ (respectively $i \leqslant n-2$ ) such that $w(i)<$ $w(i-1)<w(i+1)$ (respectively $w(i)<w(i+2)<w(i+1))$ and $w^{\prime}=w s_{i}$.

- We write $w \smile_{2}^{r} w^{\prime}$ if there exists $i \leqslant \min (r, n-1)$ such that $w(i) w(i+1)<0$ and $w^{\prime}=w s_{i}$. The relation $\smile_{2}^{0}$ never occurs.

- We write $w \smile_{3}^{r} w^{\prime}$ if $w \in \mathcal{E}_{n}^{(r)}$ and $w^{\prime}=w t$. If $r \geqslant n-1$, the relation $\smile_{3}^{r}$ never occurs.

Remark If $w \smile_{2}^{r} w^{\prime}$, then $w \smile_{2}^{r+1} w^{\prime}$. If $w \smile_{3}^{r} w^{\prime}$, then $w \smile_{3}^{r-1} w^{\prime}$ (indeed, $\mathcal{E}_{n}^{(r)} \subseteq$ $\left.\mathcal{E}_{n}^{(r-1)}\right)$.

Taskin's Theorem. With the above notation, we have:

(a) The relation $\sim_{R}^{r}$ is the equivalence relation generated by the relations $\smile_{1}, \smile_{2}^{r}$ and $\smile_{3}^{r}$.

(b) The relation $\simeq_{R}^{r}$ is the equivalence relation generated by the relations $\smile_{1}, \smile_{2}^{r}$ and $\smile_{3}^{r-1}$. 


\subsection{Proof of Theorem 1.5}

Recall that the relation $\sim_{L R}^{r}$ (respectively $\simeq_{L R}^{r}$ ) is the equivalence relation generated by $\sim_{L}^{r}$ and $\sim_{R}^{r}$ (respectively $\simeq_{L}^{r}$ and $\simeq_{R}^{r}$ ). Recall also that $x \sim_{L}^{r} y$ (respectively $x \simeq_{L}^{r} y$, respectively $x \sim_{L} y$ ) if and only if $x^{-1} \sim_{R}^{r} y^{-1}$ (respectively $x^{-1} \simeq_{R}^{r} y^{-1}$, respectively $x^{-1} \sim_{R} y^{-1}$ ). So it is sufficient to show that Theorem 1.5 holds whenever $?=R$. It is then easy to see that Theorem 1.5 will follow from Taskin's Theorem and from the following three lemmas (which will be proved in subsections 7.3, 7.4 and 7.5).

Lemma 7.1 Let $w, w^{\prime} \in W_{n}$ be such that $w \smile_{1} w^{\prime}$. Then $w \sim_{R} w^{\prime}$.

Lemma 7.2 Let $w, w^{\prime} \in W_{n}$ and let $r \geqslant 0$ be such that $b \geqslant r a$ and $w \smile_{2}^{r} w^{\prime}$. Then $w \sim_{R} w^{\prime}$.

Lemma 7.3 Let $w \in W_{n}$ and let $r \geqslant 0$ be such that $b \leqslant(r+1) a$ and $w \smile_{3}^{r} w^{\prime}$. Then $w \sim_{R} w^{\prime}$.

\subsection{Proof of Lemma 7.1}

Let $w, w^{\prime} \in W_{n}$ be such that $w \smile_{1} w^{\prime}$. Let $i \in I_{n-1}^{+}$be such that $w^{\prime}=w s_{i}$. Then $i \geqslant 2$ and $w(i)<w(i-1)<w(i+1)$, or $i \leqslant n-2$ and $w(i)<w(i+2)<w(i+1)$. In the first case, we have $w s_{i} s_{i-1}>w s_{i}>w>w s_{i-1}$ while, in the second case, we have $w s_{i} s_{i+1}>w s_{i}>w>w s_{i+1}$. So $w^{\prime}=w s_{i} \sim_{R} w$ by 1.19 and Corollary 2.2. The proof of Lemma 7.1 is complete.

\subsection{Proof of Lemma 7.2}

Let $w, w^{\prime} \in W_{n}$ and let $r \geqslant 0$ be such that $b \geqslant r a$ and $w \smile_{2}^{r} w^{\prime}$. Let $i \in I_{n-1}^{+}$be the element such that $w^{\prime}=w s_{i}$. Then $i \leqslant r$ and $w(i) w(i+1)<0$. By exchanging $w$ and $w^{\prime}$ if necessary, we may assume that $w(i)<0$ and $w(i+1)>0$.

Let us write $w=x v$, with $x \in X_{n}^{(i+1)}$ and $v \in W_{i+1}$. Then $v s_{i} \in W_{i+1}$ and $w s_{i}=$ $x v s_{i}$. Therefore, by [11, Proposition 9.11], we only need to show that $v s_{i} \sim_{L} v$. But $0<x(1)<\cdots<x(r+1)$ (see Lemma 1.4), and $v(j) \in I_{i+1}$ for all $j \in I_{i+1}$. So $v(i)>0$ and $v^{\prime}(i+1)<0$. In particular, $v \smile_{2}^{r} v s_{i}$ (and even $v \smile_{2}^{i} v^{\prime}$ ). This means that we may (and we will) assume that $i=n-1$. So we have

$$
b \geqslant(n-1) a, \quad w(n-1)<0 \quad \text { and } \quad w(n)>0,
$$

and we want to show that $w \sim_{R} w s_{n-1}$ or, in other words, that

$$
w^{-1} \sim_{L} s_{n-1} w^{-1} .
$$

Let $\alpha=\alpha_{w^{-1}}, \sigma=\sigma_{w^{-1}}$ and $\beta=\beta_{w^{-1}}$. Then

$$
w^{-1}=\alpha a_{l} \sigma a_{l} \beta^{-1} .
$$


By Lemma 1.2, there exists a unique sequence $1 \leqslant i_{1}<\cdots<i_{l} \leqslant n$ such that $\alpha a_{l}=$ $r_{i_{1}} \cdots r_{i_{l}}$ so

$$
w^{-1}=r_{i_{1}} \cdots r_{i_{l}} \sigma \beta^{-1} .
$$

But, again by Lemma 1.2, we have $w^{-1}(i)<0$ if and only if $i \in\left\{i_{1}, \ldots, i_{l}\right\}$. So

$$
i_{l}=n-1
$$

So

$$
w^{-1}=r_{i_{1}} \cdots r_{i_{l-1}} r_{n-1} \sigma \beta^{-1} .
$$

and

$$
s_{n-1} w^{-1}=r_{i_{1}} \cdots r_{i_{l-1}} r_{n} \sigma \beta^{-1} .
$$

So the result follows from Proposition 4.1.

\subsection{Proof of Lemma 7.3}

Let $w \in W_{n}$ and let $r \geqslant 0$ be such that $b \leqslant(r+1) a$ and $w^{-1} \smile_{3}^{r} w^{\prime-1}$. We want to show that $w \sim_{L} w^{\prime}=t w$. The proof goes through several steps.

First step: easy reductions. First, note that $r \leqslant n-2$. Let us write $w=v x^{-1}$, with $v \in W_{r+2}$ and $x \in X_{n}^{(r+2)}$. Then $0<x(1)<\cdots<x(r+2)$ by Lemma 1.4 , so $v^{-1} \in$ $\mathcal{E}_{r+2}^{(r)}$. Then $t w=(t v) x^{-1}$ with $t v \in W_{r+2}$ so, by [11, Proposition 9.11], it is sufficient to show that $t v \sim_{L} v$. This shows that we may (and we will) assume that $r=n-2$.

By [11, Corollary 11.7], this is equivalent to show that $t w_{n} w \sim_{L} w_{n} w$. Since $w_{n} w \in \mathcal{E}_{n}^{(n-2)}$ we may, by replacing $w$ by $t w, w_{n} w$ or $t w_{n} w$, assume that $w^{-1}(1)>$ 0 and $w^{-1}(n)>0$. Since moreover $\left|w^{-1}(1)\right|>\left|w^{-1}(i)\right|$ for all $i \in\{2,3, \ldots, n=$ $r+2\}$, we have $w^{-1}(1)=n$.

As a conclusion, we are now working under the following hypothesis:

Hypothesis. From now on, and until the end of this subsection, we assume that

(1) $w^{-1}(1)=n$ and $w^{-1}(n)>0$, and

(2) $w^{-1} \in \mathcal{E}_{n}^{(n-2)}$.

And recall that we want to show that

$$
t w \sim_{L} w
$$

Second step: decomposition of $w$. Let $v=s_{n-1} \cdots s_{2} s_{1} w$. Then $v^{-1}(n)=w^{-1}(1)=$ $n$ by (3), so $v \in W_{n-1}$. Therefore,

$$
w=s_{1} s_{2} \cdots s_{n-1} v, \quad s_{1} s_{2} \cdots s_{n-1} \in X_{n} \quad \text { and } \quad v \in W_{n-1} .
$$

Note that

$$
v^{-1}(k)=w^{-1}(k+1)
$$


for all $k \in[1, n-1]$, so that

$$
v \in \mathcal{E}_{n-1}^{(n-3)}
$$

and, by (2),

$$
v^{-1}(n-1)>0 \text {. }
$$

Let us write $v=r_{i_{1}} \cdots r_{i_{l}} \sigma \beta^{-1}$, with $l=\ell_{t}(v)=\ell_{t}(w), 1 \leqslant i_{1}<\cdots<i_{l} \leqslant n-1$, $\sigma \in \mathfrak{S}_{l, n-1-l}$ and $\beta \in Y_{l, n-1-l}$. By 7.4 and Lemma 1.2, we have

$$
i_{l} \leqslant n-2
$$

Finally, note that

$$
\sigma=\sigma_{[l+1, n-1]}
$$

Proof of 7.6 By 7.3, we have $\left|v^{-1}\left(i_{1}\right)\right|>\left|v^{-1}\left(i_{2}\right)\right|>\cdots>\left|v^{-1}\left(i_{l}\right)\right|$. Therefore, it follows from 1.14 that $\beta\left(\sigma^{-1}(l)\right)>\beta\left(\sigma^{-1}(l-1)\right)>\cdots>\beta\left(\sigma^{-1}(1)\right)$. Since $\sigma$ stabilizes the interval $[1, l]$ and since $\beta$ is increasing on $[1, l]$ (because it lies in $Y_{l, n-l}$ ), this forces $\sigma(k)=k$ for all $k \in[1, l]$.

Similarly, if $1 \leqslant j_{1}<\cdots<j_{n-l} \leqslant n$ denotes the unique sequence such that $[1, n]=\left\{i_{1}, \ldots, i_{l}\right\} \cup\left\{j_{1}, \ldots, j_{n-l}\right\}$, then $\left|v^{-1}\left(j_{1}\right)\right|>\left|v^{-1}\left(j_{2}\right)\right|>\cdots>\left|v^{-1}\left(j_{n-l}\right)\right|$ by 7.3. So it follows from 1.14 that $\beta\left(\sigma^{-1}(l+1)\right)>\beta\left(\sigma^{-1}(l+2)\right)>\cdots>$ $\beta\left(\sigma^{-1}(n)\right)$ and, since $\sigma$ stabilizes the interval $[l+1, n]$ and $\beta$ is increasing on the same interval, this forces $\sigma(l+k)=n+1-k$ for $k \in[1, n-l]$.

Third step: conclusion. We first need the following elementary result:

$$
s_{1} s_{2} \cdots s_{n-1} r_{i_{1}} \cdots r_{i_{l}}=r_{i_{1}+1} \cdots r_{i_{l}+1} s_{l+1} s_{l+2} \cdots s_{n-1} .
$$

Proof of 7.7 This follows easily from 1.2 or from 1.3. have

Now, let $\tau=s_{l+1} s_{l+2} \cdots s_{n-1} \sigma_{[l+1, n]} \beta^{-1}=\sigma_{[l+1, n]} \beta^{-1} \in \mathfrak{S}_{n}$. Then, by 7.7, we

$$
w=r_{i_{1}+1} r_{i_{2}+1} \cdots r_{i_{l}+1} \tau \text { and } \quad t w=r_{1} r_{i_{1}+1} r_{i_{2}+1} \cdots r_{i_{l}+1} \tau .
$$

By 7.5 , we have $b \geqslant\left(i_{l}+1-1\right) a$, so, by Corollary 4.4 , we have

$$
w \sim_{L} r_{2} r_{3} \cdots r_{l+1} \tau \text { and } t w \sim_{L} r_{1} r_{2} \cdots r_{l+1} \tau
$$

So we only need to show that $r_{2} r_{3} \cdots r_{l+1} \tau \sim_{L} r_{1} r_{2} \cdots r_{l+1} \tau=t r_{2} r_{3} \cdots r_{l+1} \tau$. But $r_{2} \cdots r_{l+1} \sigma_{[l+1, n]} \beta^{-1} s_{1} \cdots s_{n-1} a_{l} \sigma_{[l+1, n-1]} \beta^{-1}$, with $\beta \in Y_{l, n-1-l}$. So the result follows from Corollary 6.2.

The proof of Lemma 7.3 is complete, as well as the proof of Theorem 1.5.

Acknowledgements Part of this work was done while the author stayed at the MSRI during the winter 2008. The author wishes to thank the Institute for its hospitality and the organizers of the two programs of that period for their invitation.

The author also wants to thank warmly L. Iancu and N. Jacon for their careful reading of a preliminary version of this paper and for their useful comments. 


\section{References}

1. Bonnafé, C.: Two-sided cells in type $B$ (asymptotic case). J. Algebra 304, 216-236 (2006)

2. Bonnafé, C.: Semicontinuity properties of Kazhdan-Lusztig cells. Preprint (2008), available at arXiv: 0808.3522

3. Bonnafé, C., Geck, M., Iancu, L., Lam, T.: On domino insertion and Kazhdan-Lusztig cells in type $B_{n}$. Progress in Mathematics (2009, to appear), available at arXiv:math/0609279

4. Bonnafé, C., Iancu, L.: Left cells in type $B_{n}$ with unequal parameters. Represent. Theory 7, 587-609 (2003)

5. Geck, M.: On the induction of Kazhdan-Lusztig cells. Bull. London Math. Soc. 35, 608-614 (2003)

6. Geck, M.: Computing Kazhdan-Lusztig cells for unequal. J. Algebra 281, 342-365 (2004)

7. Geck, M., Pfeiffer, G.: Characters of Finite Coxeter Groups and Iwahori-Hecke Algebras. London Math. Soc. Monographs, New Series, vol. 21. Oxford University Press, London (2000)

8. Kazhdan, D.A., Lusztig, G.: Representations of Coxeter groups and Hecke algebras. Invent. Math. 53, 165-184 (1979)

9. Lam, T.: Growth diagrams, domino insertion, and sign-imbalance. J. Comb. Theory Ser. A. 107, 87115 (2004)

10. Lusztig, G.: Left cells in Weyl groups. In: Herb, R.L.R., Rosenberg, J. (eds.) Lie Group Representations I. Lecture Notes in Math., vol. 1024, pp. 99-111. Springer, Berlin (1983)

11. Lusztig, G.: Hecke Algebras with Unequal Parameters. CRM Monograph Series, vol. 18. American Mathematical Society, Providence (2003). 136 p.

12. Pietraho, T.: Knuth relations for the hyperoctahedral groups. J. Algebraic Combin. (2008, to appear), available at arXiv:0803.3335

13. Pietraho, T.: Module structure of cells in unequal parameter Hecke Algebras. Nagoya Math. J. (2009, to appear), available at arXiv:0902.1907

14. Taskin, M.: Plactic relations for $r$-domino tableaux. Preprint (2008), available at arXiv:0803.1148

15. van Leeuwen, M.: The Robinson-Schensted and Schutzenberger algorithms, an elementary approach, The Foata Festschrift. Electron. J. Combin. 3 (1996), Research Paper 15

16. Shimozono, M., White, D.E.: Color-to-spin ribbon Schensted algorithms. Discrete Math. 246, 295316 (2002) 